INRA Prod. Anim., $2005,18(5), 295-308$

\title{
Le canard mulard : déterminisme génétique d'un hybride intergénérique
}

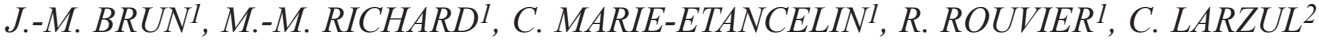 \\ ${ }^{1}$ INRA, Station d'Amélioration Génétique des Animaux, F-31326 Castanet-Tolosan Cedex \\ ${ }^{2}$ INRA, Station de Génétique Quantitative et Appliquée, F-78352 Jouy-en-Josas Cedex
}

Courriel :brun@germinal.toulouse.inra.fr

La maîtrise de l'insémination artificielle dans l'hybridation entre le canard de Barbarie et la cane commune a conduit, au cours de ces 20 dernières années, à la suprématie du mulard comme producteur de foie gras. Quels sont donc les atouts de cet hybride ? Que peut-on encore attendre de son amélioration génétique?

Avec une production de $16400 \mathrm{~T}$ de foie gras en 2003 (soit $83 \%$ de la production mondiale), la France domine le marché international $\mathrm{du}$ foie gras (Pé 2004). En France, trois types de palmipèdes sont gavés pour produire du foie gras : l'oie (les deux sexes), le canard de Barbarie et le canard mulard mâles. Le mulard est un hybride infertile issu d'un croisement intergénérique entre un canard de Barbarie et une cane commune. En 2004, le canard mulard a fourni à lui seul plus de $90 \%$ du foie gras produit en France. Il assure, en outre, une production de produits carnés originaux, comme les magrets (filets de canards gavés) et les confits.
La production de foie gras de mulard a été multipliée par cinq au cours de ces 20 dernières années (1985-2005), alors que celle des autres palmipèdes gavés a régressé durant la même période. En Asie du sud-est, les mulards mâle et femelle sont utilisés pour la production de canard à rôtir. Le mulard est connu en France depuis le XVI ${ }^{\mathrm{e}}$ siècle, date de l'introduction du canard de Barbarie, et son intérêt pour la production de foie gras est documenté depuis le XVIII siècle (Duhart 2004). En effet, les qualités du canard mulard sont largement reconnues : rusticité en élevage, facilité de gavage, foie gras de poids élevé et de bonne qualité (Guy et

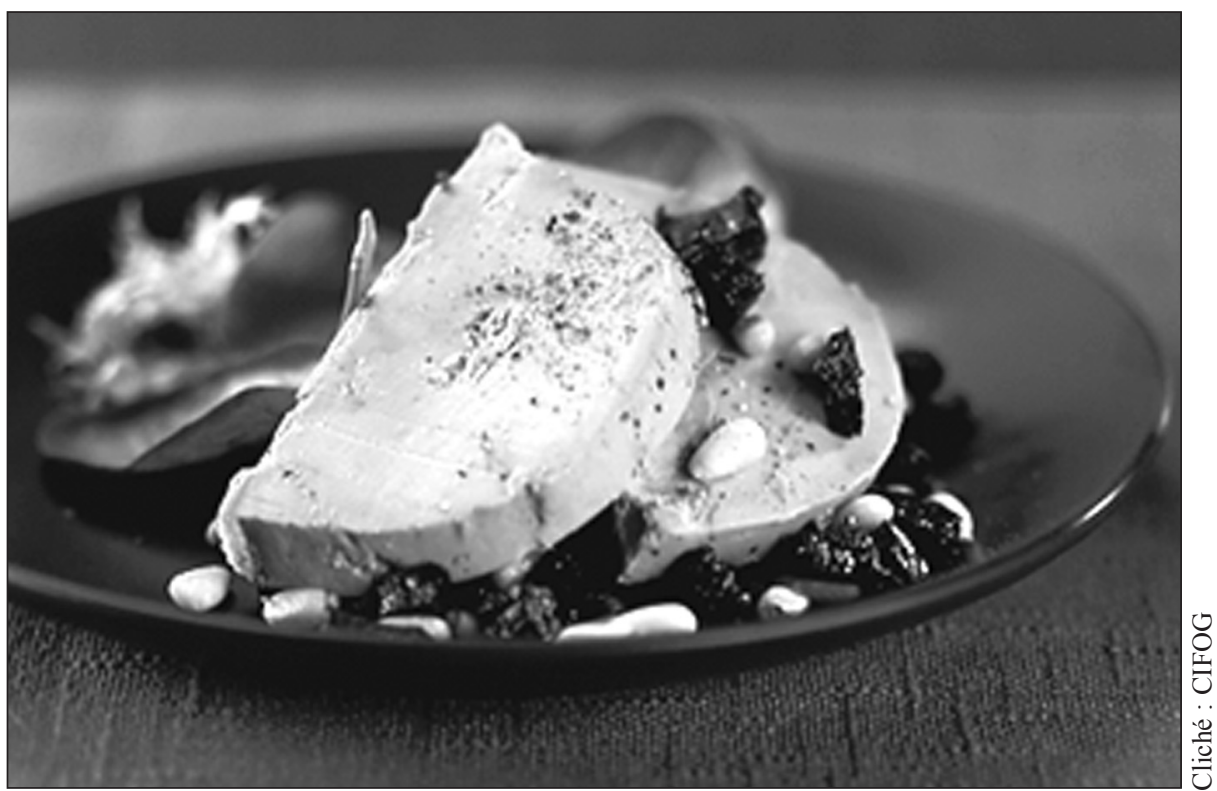

Actuellement, $90 \%$ du foie gras produit en France est issu du canard mulard al 1995). Jusqu'à la fin des années 80, le handicap majeur résidait dans la difficulté d'obtenir des mulards par accouplement naturel. Sa production doit d'abord son essor au développement de l'insémination artificielle, mais aussi aux progrès réalisés dans la maîtrise des conditions d'incubation artificielle et à l'amélioration des techniques de gavage.

L'objectif de cet article est de faire le point des connaissances d'ordre génétique relatives au canard mulard. Il s'agit d'abord de décrire les «spécificités» de cet hybride, relativement aux espèces parentales, puis de faire une synthèse sur les paramètres génétiques qui vont orienter les stratégies d'amélioration génétique. L'amélioration génétique classique est basée à la fois sur le choix de souches et de leurs croisements, et sur la sélection intra-souche. L'utilisation optimale des souches en croisement repose sur la connaissance des effets génétiques en croisement, effets additifs directs et maternels, effets d'hétérosis, encore appelés paramètres du croisement. L'optimisation de la sélection suppose de connaître les valeurs des héritabilités et des corrélations génétiques des caractères à sélectionner. L'originalité de la situation du mulard, hybride stérile, est que la sélection doit se faire dans chacune des deux populations parentes. Les corrélations génétiques pur-croisement sont alors importantes à considérer. De nouvelles possibilités d'amélioration génétique sont offertes par le développement de la 
génétique moléculaire, au travers de marqueurs moléculaires des caractères d'intérêt. Ces connaissances nouvelles en génétique moléculaire seront également synthétisées dans le présent article.

Les caractères d'intérêt économique concernent la reproduction de la cane commune mère du mulard, la croissance et l'efficacité alimentaire, la composition corporelle, l'aptitude au gavage et la qualité des produits, foie et viande, et enfin l'adaptation des mulards aux conditions d'élevage et de gavage.

\section{1 / Eléments de zoologie}

Le canard mulard est un hybride intergénérique : son père, le canard de Barbarie (Cairina moschata) et sa mère, une cane commune (Anas platyrhynchos) appartiennent à deux genres différents. Le croisement réciproque est également possible et l'hybride qui en résulte est appelé «hinny» ou «bardeau». Cairina moschata est un oiseau percheur originaire d'Amérique du sud. Anas platyrhynchos est un oiseau barboteur, dont le variant sauvage est le canard colvert. Ces deux espèces sont génétiquement isolées depuis le début du miocène, soit 50 millions d'années.

\section{1 / Cytogénétique}

Comme pour la plupart des espèces avicoles, les chromosomes des canards se répartissent en deux classes de taille, les macro et les microchromosomes, chromosomes de type ancestral. De plus, autre spécificité des volailles, au niveau des chromosomes sexuels, c'est le sexe femelle qui est hétérogamétique (femelle $=$ ZW, mâle $=$ ZZ).

Les études en cytogénétique sur les chromosomes de canard n'ont pas encore abouti à une standardisation, ne serait-ce qu'au niveau des macrochromosomes. Il semble maintenant avéré que le nombre de chromosomes (micro et macro) soit de $2 \mathrm{~N}=80$ tant chez le canard commun que chez le canard de Barbarie (Mott et al 1968, Tesoro et al 1986, Denjean et al 1997, Vinoo et al 1999). Les différences observées entre les deux genres Anas et Cairina se situent au niveau des chromosomes sexuels et des macrochromosomes 2, 3, 5, 7 ou 11 (les différences notées au niveau des chromosomes 7 dans une étude et 11 dans une autre étude peuvent sans doute être attribuées à la même paire de chromosomes qui a été numérotée de manière différente dans les deux études, Denjean et al 1997). Pour le chromosome 2, la taille du bras court est supérieure chez le canard commun. Pour les chromosomes 3 et 5 , le canard de Barbarie possède une bande supplémentaire dans les régions 2 et $2-1$ du bras q, respectivement. Enfin, au niveau du chromosome 7 (ou 11), seul le centromère est hétérochromatique chez le canard de Barbarie, alors que l'ensemble du chromosome est hétérochromatique chez le canard commun.

\section{2 / Les souches utilisées en pro- duction de mulards}

Le canard «commun»a été domestiqué en Chine, il y environ 4000 ans, à partir du canard colvert. Il en existe aujourd'hui un grand nombre de races, de formats et d'aptitudes différents. Les races utilisées dans le sud-est asiatique pour la production d'œufs de consommation sont très pondeuses et de petit format, comme la cane Tsaiya ou le coureur indien. Les races utilisées pour la production de viande sont plus lourdes. La race Pékin, au plumage blanc,

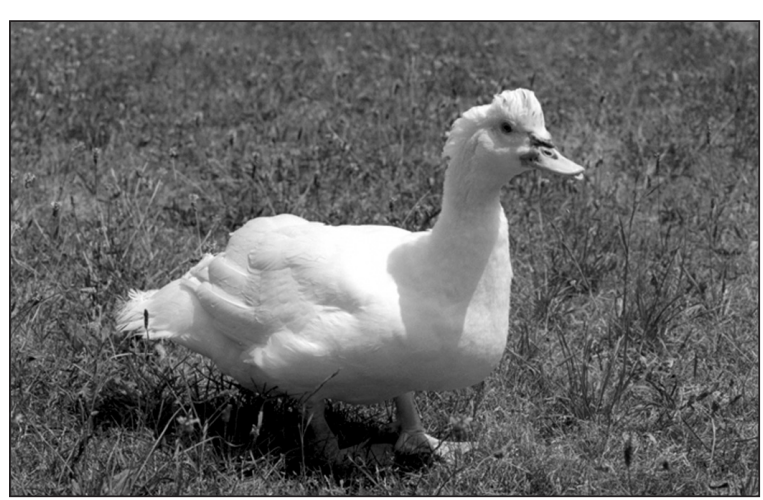

Canard de Barbarie
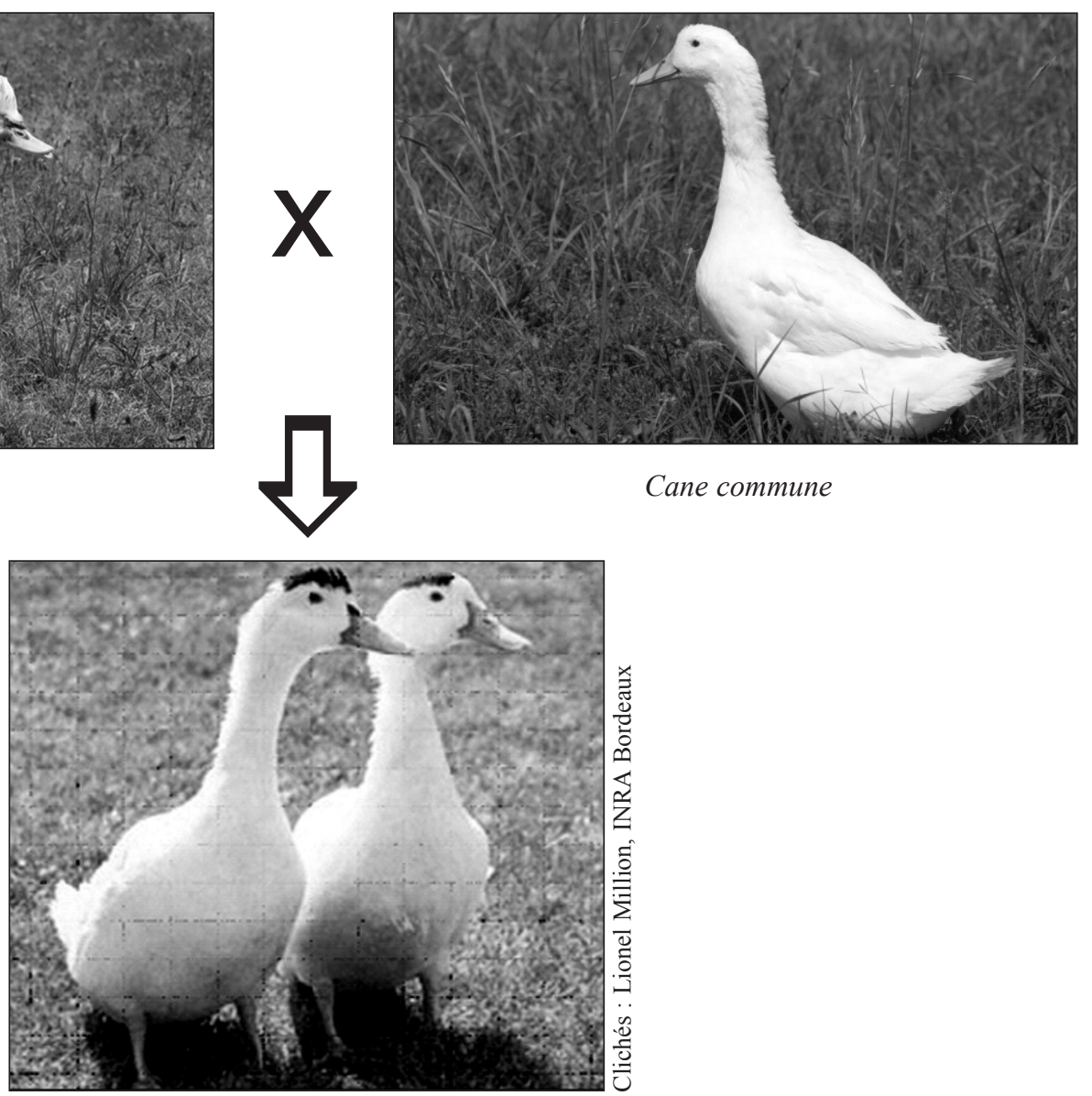

Canard mulard 
importée de Chine vers 1870, est aujourd'hui la plus utilisée en France pour la production de mulards, supplantant la race Rouen (plumage proche de celui du colvert). Les canes Pékin utilisées comme mères du mulard, de poids adulte compris entre 4 et $5 \mathrm{~kg}$, sont généralement des canes croisées issues de lignées sélectionnées respectivement pour le poids corporel et la production d'œufs, parfois sur la fertilité. Certaines souches ont été sélectionnées pour la couleur blanche de leurs mulards (on parle de «blanchiment»). Il existe également des lignées synthétiques de format moyen issues de croisements entre les races Tsaiya et Pékin, telle que la souche expérimentale INRA44. Chez le canard de Barbarie, il n'y a pas de races identifiées. Il existe différentes souches utilisées pour la production de mulard qui présentent une diversité de coloration (Migliore et al 1988) et de poids adulte, le poids adulte des mâles variant, selon la souche entre 5 et $6 \mathrm{~kg}$.

\section{3 / Accouplement et insémina- tion}

L'accouplement spontané entre un mâle Barbarie et une cane commune est rare, ce qui peut être attribué à une différence de comportement : le mâle Barbarie est un animal peu mobile alors que la cane commune est agile et semble réticente à ce type d'accouplement. Il s'ensuit que seulement 15 à $45 \%$ des œufs pondus en régime d'accouplement naturel sont fertiles (Watanabe 1961, Olver et al 1977, Hoffmann 1992).

L'accouplement naturel peut cependant être favorisé par l'homme, comme à Taïwan, où les fermiers intervenaient dans les mares deux fois par semaine pour immobiliser les canes et permettre la saillie. Cela peut se réaliser aussi en constituant des petits groupes de reproduction de 2 ou 3 mâles et d'une dizaine de canes. Lorsque la taille du groupe augmente et que plus de 10 mâles Barbarie se retrouvent dans le même parc, des combats territoriaux se produisent, les mâles montrent une activité homosexuelle et délaissent les canes, la fertilité diminue (Gvaryahu et al 1984). Watanabe (1961) a réalisé les premières recherches sur l'insémination artificielle. Huang et Chow (1974) ont montré l'influence de l'intervalle entre deux inséminations successives sur le taux de fertilité. Les avancées majeures ont été proposées par Tai Liu et Tai (1984, 1991) qui ont mis au point la technique de collecte de la semence et d'insémination artificielle (IA). Ces travaux ont permis le développement et la généralisation de l'IA. Bien maîtrisée et pratiquée deux fois par semaine, elle conduit à des taux de fertilité variant entre 65 et $80 \%$.

\section{4 / Durée de la période fertile}

Une caractéristique remarquable de la reproduction des oiseaux est que la femelle continue à pondre des œufs fertiles pendant plusieurs jours après une insémination naturelle ou artificielle. Sa base physiologique est le stockage des spermatozoïdes dans des glandes spécialisées du tractus génital de la femelle, les glandes utéro-vaginales. Ces spermatozoïdes sont ensuite progressivement relargués et remontent dans l'oviducte pour aller féconder les ovules pondus par l'ovaire dans l'infundibulum (Brillard 1993, Etches 1996). Cela détermine une certaine «durée de la fertilité», dont l'un des critères est la durée maximale de la période fertile, nombre de jours entre l'insémination et le dernier œuf fécondé (Lake 1975). Sa valeur moyenne est caractéristique de chaque espèce. Dans le cas du croisement intergénérique qui conduit au mulard, cette durée est faible, ce qui oblige à inséminer les femelles deux fois par semaine pour maintenir un taux de fertilité acceptable.

\section{5 / Développement embryon- naire}

Les œufs avec embryon mulard nécessitent 32 jours d'incubation, ce qui se situe à la moyenne des durées d'incubation des espèces parentales, 28 jours chez le canard commun et 35 jours chez le Barbarie. Les œufs avec embryon hinny nécessitent 34 jours d'incubation.

Rapporté pour la première fois par Ramirez et al (1976), le déséquilibre du sex-ratio à la naissance, en faveur des mulards mâles, est vérifié en permanence par les accouveurs qui pratiquent le sexage à l'éclosion. Ce rapport est d'environ 55 mâles pour 100 canetons nés. Le croisement réciproque présente un déséquilibre similaire en faveur des mâles (Batellier et al 2004). Ce déséquilibre est conforme à la règle de Haldane (1922) qui postule que le déficit de viabilité ou de fertilité d'un hybride interspécifique concerne le sexe hétérogamétique, en l'occurrence la femelle. D'après Batellier et al (2004), le déséquilibre du sex-ratio serait dû à un excès de mortalité intermédiaire et tardive des embryons femelles, survenant entre le $5^{\mathrm{e}}$ jour d'incubation, où aucun déséquilibre n'est mis en évidence, et l'éclosion. Cependant, les effectifs relativement faibles d'embryons sexés au $1^{\mathrm{er}}$ et au $5^{\mathrm{e}}$ jour d'incubation dans cette expérience ne permettent pas d'exclure complètement l'hypothèse d'une installation précoce de ce déséquilibre.

\section{6 / Stérilité des hybrides}

Les mulards mâles et femelles sont stériles. Les mâles ont des testicules normalement développés, des niveaux élevés de testostérone plasmatique et présentent une activité sexuelle (Snapir et al 1998) mais ne produisent pas de spermatozoïdes. Leur spermatogénèse s'arrête à la prophase méïotique, au stade spermatocyte primaire (Bebak 1959, Gomot 1973, Huang et Sung 1988, Snapir et al 1998). Si les testicules du mulard mâle sont normalement développés, les ovaires de la femelle mularde restent rudimentaires. Ses follicules, lorsqu'ils existent, dégénèrent progressivement sans qu'il y ait ponte ovulaire et l'oviducte a un diamètre très réduit (Gomot et Bonin 1965).

Chez la femelle hinny, au contraire, l'ovaire possède des ovocytes normaux, qui donnent des ovules pendant les premières années de vie de l'animal (Lutz-Ostertag 1965). Le hinny mâle produit des spermatozoïdes (Marchand et Gomot 1972).

L'infertilité du mulard pourrait s'expliquer par une impossibilité d'appariement des chromosomes provenant des deux espèces parentales au moment de la meïose dans les cellules germinales de l'hybride. Il s'agit de stérilité chromosomique, alors que la stérilité génétique implique des loci où les allèles des espèces parentales conduisent à des dysharmonies de développement et stérilité.

\section{2 / Reproduction de la cane commune et production de canards mulards}

Le nombre de mulards produits par cane dans son cycle de ponte dépend non seulement de sa production d'œufs incubables, mais aussi de leur fécondation, donc de la fertilité de la cane, et ensuite de l'éclosabilité des œufs fécondés. Cette dernière est conditionnée par la viabilité embryonnaire entre la fécondation et l'éclosion.

\section{1 / Performances de ponte}

Dans des conditions de production intensives, la ponte d'une cane de race Pékin peut être initiée dès l'âge de 
22 semaines et persister pendant 40 semaines, conduisant à un nombre d'œufs pondus de l'ordre de 180 (Sauveur 1988). La cane est généralement exploitée sur un seul cycle de ponte. Les performances de ponte dépendent de la race et du mode de conduite. La race Tsaiya Brune, une race de canes pondeuses de petit format élevée à Taiwan $(1,4 \mathrm{~kg}$ de poids adulte) peut pondre jusqu'à 337 œufs à l'âge de 500 jours (Chen 1992).

Le croisement entre races de canards communs apporte généralement une augmentation de la ponte par rapport à la moyenne des races parentales. Ainsi, Velez et al (1996) estiment un effet d'hétérosis de $10 \%$ sur le nombre d'œufs pondus à l'âge de 52 semaines dans le croisement entre les races Tsaiya Brune et Pékin européenne. Hetzel $(1983,1984)$ rapporte des valeurs de $7 \%$ entre les races indonésiennes Alabio et Tegal et de $23 \%$ entre les races Alabio et Khaki Campbell.

Une revue de Pingel (1990a) et quelques publications récentes (Cheng et al 1995, Brun et Larzul 2003) sur l'héritabilité du nombre d'œufs pondus chez la cane Pékin $\left(\mathrm{h}^{2}\right.$ variant entre 0,17 et 0,32 ) indiquent que ce caractère peut être amélioré par sélection. Les mêmes sources indiquent une héritabilité élevée de l'âge au premier œuf $\left(h^{2}=0,34\right.$ et 0,47$)$, composante importante du nombre d'œufs pondus.

\section{2 / Fertilité et durée de la période fertile}

Le taux de fertilité est le pourcentage d'œufs fécondés par rapport aux œufs mis en incubation. Si ce caractère fait intervenir les deux parents, il est souvent rapporté à la cane. La fertilité est évaluée par un mirage des œufs, réalisé généralement à partir du 7e jour d'incubation, permettant de distinguer les œufs apparemment non fécondés des œufs ayant donné lieu à un développement embryonnaire. Une mortalité embryonnaire très précoce et indétectable au mirage conduit à une sous-estimation de la fertilité réelle. Un examen plus approfondi du disque germinatif après ouverture de l'œuf permet d'affiner le diagnostic de la fertilité dans les études expérimentales.

Dans la suite, nous distinguerons la fertilité obtenue dans le rythme conventionnel de deux IA par semaine et la fertilité après une seule IA, permettant de mesurer la durée maximale de la période fertile. Sur un plan appli- qué, les études sur la durée maximale de la période fertile trouvent leur motivation dans le fait qu'un allongement de celle-ci permettrait d'inséminer une seule fois par semaine au lieu de deux.

\subsection{1 / Fertilité avec deux IA par semaine}

Sur 220000 œufs de canes Kaiya (métisses Tsaiya x Pékin) inséminées avec de la semence mélangée de mâles Barbarie, issus de 3 élevages Taiwanais, Rouvier et al (1984) trouvent une fertilité variant de 63 à $83 \%$ avec une moyenne de $71 \%$. Inséminées avec de la semence de canard commun (en souche pure), la fertilité des canes communes est de l'ordre de $90 \%$. Les études comparatives des deux systèmes de reproduction sont rares : Brun et Larzul (2003) trouvent un taux de fertilité en croisement de $66,4 \%$ sur des canes expérimentales INRA44 (mirage au $12^{\mathrm{e}}$ jour d'incubation) et de $85,5 \%$ sur les mêmes canes inséminées en souche pure. Cet écart est confirmé par Brun et al (2004), si l'on prend comme indicateur de la fertilité en deux IA par semaine, la fertilité estimée sur les trois premiers jours suivant l'IA, on trouve des taux de fertilité de $89,6 \%$ en pur et $69,9 \%$ en croisement.

Ces différences de fertilité purcroisement s'expliquent en partie par des différences dans le nombre de spermatozoïdes qui atteignent le site de la fécondation, selon leur type génétique, estimé par le nombre de spermatozoïdes péri-vitellins (Sellier et al 2005). Parmi les mécanismes en cause, on peut invoquer l'hypothèse de limites au transport des spermatozoïdes dans le vagin, de nature spécifique, dont l'existence est établie chez la poule (Steele et Wishart 1992).

Le taux de fertilité correspondant aux quatre types d'accouplement entre le canard commun et le canard de Barbarie a été estimé par Brun et al (1999) à 72,3\% (commun x commun), $69,8 \%$ (Barbarie x Barbarie), 44,0\% (Barbarie x commun pour la production de mulards) et $34,3 \%$ (commun $x$ Barbarie pour la production de hinny) sur la base d'un mirage des œufs. Corrigées après autopsie des œufs apparemment non fécondés, ces estimations sont de $77,9 \%, 76,0 \%, 47,8 \%$ et $45,9 \%$ pour ces 4 types d'accouplements respectivement. Ces chiffres indiquent des taux de fertilité identiques dans les deux espèces parentales, et supérieurs à ceux des deux croisements intergénériques. Obtenus en une IA par semaine, ces résultats traduisent des différences de niveau de fertilité initiale et de durée de la fertilité sur 7 jours. Par ailleurs, ces résultats correspondent aux seules inséminations ayant donné au moins un œuf fécondé. Le pourcentage de ces inséminations fructueuses par rapport à l'ensemble des inséminations est estimé à 94,5\%, $88,4 \%, 72,0 \%$ et $45,8 \%$ dans ces quatre types d'accouplements, indiquant la difficulté d'obtenir des hinnies par insémination.

Dans le croisement pour produire le mulard, le taux de fertilité peut dépendre de la race (ou de la souche) de la cane, de celle du mâle Barbarie et enfin de leur interaction (Mialon 1985). En ce qui concerne les variations liées au type génétique de la cane, Velez et al (1996), étudiant le croisement entre les souches de canes Tsaiya Brune et Pékin, ont mis en évidence des différences d'effets additifs directs et maternels entre ces souches. La cane Tsaiya transmet à ses filles des gènes qui ont un effet favorable sur leur fertilité (effets directs) mais possède des gènes qui exercent un effet maternel défavorable. Le bilan reste en faveur de la souche Tsaiya dont le taux de fertilité, cumul de ses effets directs et maternels, est de $75,9 \%$ vs $71,3 \%$ pour la Pékin. Un effet d'hétérosis relativement faible et non significatif $(5,4 \%)$ est observé sur le taux de fertilité des canes croisées.

Brun et Larzul (2003) estiment à 0,36 l'héritabilité du taux de fertilité en 2 IA par semaine de canes INRA44 inséminées en croisement. Cette valeur élevée traduit un potentiel important de progrès génétique par sélection. L'héritabilité de la fertilité obtenue en souche pure dans la même population est significativement plus faible $\left(\mathrm{h}^{2}=0,15\right)$. Au vu de sa forte corrélation génétique avec le nombre de mulards éclos $\left(\mathrm{r}_{\mathrm{g}}=0,73\right)$, le taux de fertilité en croisement apparaît comme un facteur déterminant du résultat à l'éclosion. Sa corrélation génétique avec la ponte, faible mais positive, est favorable à la sélection. La corrélation génétique entre la fertilité en croisement et la fertilité en pur est estimée à 0,50 (Brun et Larzul 2003). Cette valeur peu élevée indique que ce sont des gènes en partie différents qui contrôlent les deux caractères. Du point de vue de la sélection de la fertilité en croisement dans ce rythme de deux IA par semaine, ces résultats indiquent qu'il vaut mieux sélectionner directement sur la fertilité en croisement. 
Figure 1. Evolution du taux de fertilisation des œufs en fonction du délai après insémination obtenu sur des canes communes INRA44 inséminées avec de la semence de mâles communs (pur) ou avec de la semence de mâles Barbarie (croisement) (Brun et al 2004).

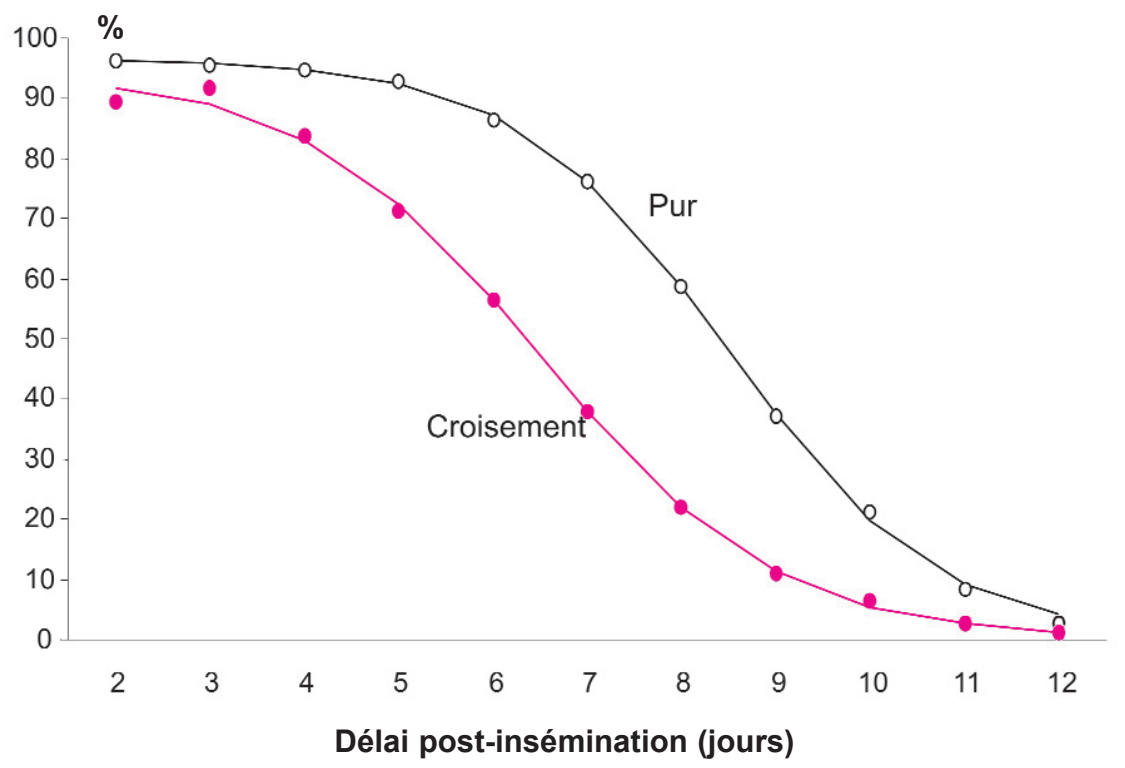

\subsection{2 / Durée de la période fertile}

La figure 1 illustre, pour des canes communes INRA44, que la diminution de la fertilité apparente dans les jours qui suivent l'insémination intervient plus vite lorsque les canes sont inséminées avec de la semence de Barbarie (en croisement) que lors d'inséminations en souche pure. En d'autres termes, la durée de la fertilité y est moins longue. En moyenne, la durée maximale de la période fertile $(\mathrm{Dm})$ est estimée à 6,4 jours en croisement et à 8,1 jours en pur. On observe une extinction quasi complète de la fertilité au $14 \mathrm{e}$ jour après l'IA, en pur comme en croisement. La décroissance rapide de la fertilité à partir du $5^{\mathrm{e}}$ jour après IA a également été notée par Tai et al (1994) sur des canes Tsaiya Brune. Dans le cas de la figure 1 , le taux de fertilisation moyen des œufs sur les 11 jours suivant l'IA est de $61,1 \%$ en pur et de $42,8 \%$ en croisement. L'âge de la cane influence Dm (Brun et al 2004), tout particulièrement en pur, avec de meilleures performances vers l'âge de 40 semaines $(\mathrm{Dm}=$ $8,2 \mathrm{j})$ par rapport à celui de 50 semaines $(\mathrm{Dm}=7,6 \mathrm{j})$.

Les études génétiques de la durée de la période fertile ont concerné jusqu'à présent la souche maternelle du mulard. Tai et al (1994) ont estimé l'héritabilité de différents critères de durée de la fertilité et indiqué que le meilleur critère de sélection de la durée de la fertilité semblait être le nombre d'œufs fécondés du $2^{\mathrm{e}}$ au $15^{\mathrm{e}}$ jour après l'IA $\left(\mathrm{h}^{2}=0,29\right)$. Dans une souche de Tsaiya Brune sélectionnée sur ce critère,
Poivey et al (2001) estiment l'héritabilité du nombre d'œufs fécondés après une seule insémination à 0,30 et celle de la durée maximale de la période fertile à 0,28 . Cheng et al (2002) démontrent clairement l'efficacité de cette sélection pour augmenter le nombre d'œufs fécondés après une seule insémination (gain de 2,55 en 7 générations pour une valeur initiale de 4,23), avec une réponse corrélée sur la durée maximale de la période fertile (gain de 2,9 jours par rapport à une valeur initiale de 5,6 j) et sans conséquences négatives sur la viabilité embryonnaire. Cette expérience montre que l'objectif d'atteindre, par sélection de la cane, un taux de fertilité de $75 \%$ en une seule IA par semaine est réaliste.

Dans la souche INRA44, Brun et al (2002) estiment l'héritabilité de Dm en croisement à 0,17 , valeur inférieure à celle citée précédemment. Dans cette souche, la durée de la fertilité en croisement présente une corrélation génétique élevée avec la durée de la fertilité en souche pure $\left(r_{g}=0,82\right)$. Les gènes qui contrôlent la durée de la fertilité en croisement et ceux qui contrôlent ce même caractère en pur sont donc largement communs. Comme l'héritabilité de la durée de la fertilité est trouvée supérieure en souche pure $\left(\mathrm{h}^{2}=0,27\right.$ contre 0,17 en croisement, Brun et al 2002), il peut être plus efficace de sélectionner en souche pure en vue d'obtenir une réponse indirecte en croisement. Ce résultat est valable dans le cas de la souche INRA44, sachant que le choix d'un critère de sélection dépend de la population à sélectionner.

\section{3 / Eclosabilité des œufs ferti- les/mortalité embryonnaire}

L'éclosabilité est le pourcentage d'œufs fertiles qui poursuivent leur développement jusqu'à l'éclosion d'un caneton vivant. Le complément à 100 de l'éclosabilité représente le pourcentage de mortalité embryonnaire. L'éclosabilité des œufs mulards est généralement trouvée de l'ordre de 70 à $80 \%$ des œufs fertiles (Watanabe 1959, Rouvier et al 1988, Cheng et al 2002). Il y a peu d'estimations comparatives du taux d'éclosabilité entre embryons mulards et embryons de souche pure issus de la même population de canes. Brun et Larzul (2003) obtiennent des valeurs similaires $(71,6 \%$ en croisement et $70,6 \%$ en pur, en pourcentage des embryons vivants au $12^{\mathrm{e}}$ jour d'incubation). Estimé dans le contexte de l'étude de la durée de la fertilité de canes INRA44 (11 jours de récolte après une seule IA), ce paramètre s'établit à $71,0 \%$ des aufs fécondés en croisement contre 75,4\% en pur (Brun et al 2004).

La mortalité globale entre la fécondation et l'éclosion doit être analysée en fonction du moment où elle se produit car les causes de mortalité diffèrent selon le stade (Sauveur 1988). On distingue la mortalité précoce, survenant entre la fécondation et le $1^{\mathrm{er}}$ mirage (7e jour d'incubation), la mortalité intermédiaire, entre le $7 \mathrm{e}$ jour d'incubation et le $2^{\mathrm{e}}$ mirage qui précède la mise en éclosoir, et la mortalité à l'éclosoir, qualifiée de tardive. Brun et al (2004) trouvent une mortalité précoce apparente (estimée par mirage) de l'ordre de $2,5 \%$ des œufs fécondés et ne différant pas entre embryons mulards et embryons de souche pure. Ils notent une différence faible mais significative de mortalité intermédiaire $(6,3 \%$ des embryons vivants au $1^{\text {er }}$ mirage en mulards $v s 3,9 \%$ en pur), mais aucune différence de mortalité à l'éclosoir.

Si le mirage ne révèle pas de différences de mortalité embryonnaire précoce entre embryons mulards et embryons «communs», l'autopsie des œufs apparemment clairs au $6^{\mathrm{e}}$ jour d'incubation indique, en revanche, une différence de mortalité embryonnaire très précoce, survenant avant même l'oviposition, estimée en mulard à $11,1 \%$ des œufs fécondés et à 5,6\% en pur (Sellier et al 2005). Au total, la mortalité précoce s'élève à $14,9 \%$ des œufs fécondés en croisement et à 10,3\% en pur (Brun et al 2004). La valeur obtenue en croisement se situe dans la fourchette estimée par Hu (1999), après ouverture et ob- 
servation à l'œil nu des œufs apparemment clairs, de $9,4-19,1 \%$. Selon Tchelycheva et al (1993), ce surcroît de mortalité précoce chez les embryons hybrides pourrait en partie s'expliquer par un blocage de la condensation de certaines régions chromosomiques lors des mitoses, blocage observé spécifiquement chez les mulards. Cependant, les différences de mortalité précoce contribuent peu à l'écart de fertilité apparente entre reproduction en souche pure et en croisement, qui reste donc majoritairement liée à des différences de taux de fertilisation des œufs (Brun et al 2004).

Brun et Larzul (2003) trouvent une héritabilité de 0,32 pour le taux d'éclosabilité des œufs à mulards de canes communes INRA44. Ce caractère présente une corrélation génétique forte $\left(r_{g}=0,88\right)$ avec le taux d'éclosabilité mesuré sur les mêmes canes inséminées en souche pure, indiquant que les gènes de la cane qui déterminent l'éclosabilité ne sont pas spécifiques du type génétique de l'embryon, qu'il soit pur ou croisé, mais déterminent en fait une aptitude générale à l'éclosabilité. La corrélation génétique entre éclosabilité et fertilité est faible $\left(r_{g}=0,37\right.$ en pur, $\mathrm{r}_{\mathrm{g}}=0,01$ en croisement). En outre, la corrélation génétique de 0,36 trouvée entre l'éclosabilité des œufs à mulards et le poids des mulards à l'âge de 6 semaines suggère une liaison positive entre la viabilité des embryons mulards et leur croissance.

La viabilité embryonnaire mérite d'être améliorée pour diminuer la perte d'œufs fécondés. Les valeurs trouvées pour l'héritabilité de ce caractère montrent qu'une sélection de la cane commune pour l'améliorer est possible, aussi bien pour des embryons de souche pure que pour des embryons mulards.

\section{3 / Caractéristiques du canard mulard}

En France, le canard mulard est essentiellement élevé pour la production de foie gras, la carcasse étant également valorisée sous la forme de pièces de découpe (magret, cuisse). La valorisation de la carcasse a conduit à prendre en compte non seulement les performances de gavage et de poids du foie mais également le poids de la carcasse et sa composition, avec un alourdissement progressif, parallèlement à une diminution de l'âge de mise en gavage. La production de mulard uni- quement pour la chair est peu répandue en France, même si elle est envisagée pour valoriser les canes mulardes. Jusqu'à présent, pour des raisons réglementaires, celles-ci ne sont pas gavées. En revanche, la production de viande à partir de canard mulard existe dans les pays du sud-est asiatique, en concurrence du canard Pékin.

Les études sur le mulard ont porté sur une grande variété de caractères, la croissance et la composition corporelle, mais surtout l'aptitude au gavage. Plus récemment, des études ont été menées pour approfondir les mécanismes de l'engraissement du foie et apprécier la qualité des produits. Quelques études ont également porté sur le comportement du canard mulard et son adaptation aux conditions d'élevage. Les principaux résultats sont disponibles dans des comparaisons entre canards mulards et espèces parentales ou entre des canards mulards issus de différentes souches parentales. Il existe encore peu d'études qui estiment le déterminisme génétique de ces caractères, soit en terme de variabilité génétique pour orienter la sélection dans les espèces parentales, soit en terme de paramètres de croisement (effets génétiques additifs directs et maternels, hétérosis) pour comprendre l'expression de ces caractères chez le canard mulard par rapport aux espèces parentales.

\section{1 / La croissance}

En comparaison d'autres espèces aviaires domestiques, les palmipèdes ont une croissance très rapide au cours des premières semaines (Pingel 1990b). La croissance suit une courbe sigmoïde (Brody 1945), caractérisée par la croissance spécifique initiale, le poids asymptotique et l'âge au point d'inflexion (ou croissance maximale). Ces 3 paramètres de la courbe varient d'une espèce à l'autre. Chez le canard Pékin, le point d'inflexion est très précoce : entre 24,1 et 26,8 jours pour les femelles, et entre 22,5 et 27,6 jours pour les mâles (Knizetova et al 1991, Gille et Salomon 1994, Maruyama et al 1999). Il est nettement plus tardif chez le canard de Barbarie : 30 jours pour les femelles et entre 34,6 et 37,2 jours pour les mâles (Leclercq 1990, Baéza et al 1997). La croissance rapide des canards communs permet un développement précoce des plumes et des tissus gras, compatible avec le mode de vie aquatique des canards colvert, ancêtre des souches domestiques de canards communs (Knizetova et al 1995). Les canards de Barbarie, ayant un mode de vie percheur ont une croissance moins précoce avec un développement préférentiel des organes locomoteurs. Chez les mulards, la croissance est intermédiaire entre celle des deux espèces parentales, avec un point d'inflexion situé entre 25 et 30,5 jours pour les mâles (Gille et Salomon 1994, Setiawan et al 1994).

Sans analyser et modéliser l'ensemble de la courbe de croissance, plusieurs études ont porté sur la croissance des canards mulards jusqu'à l'âge d'abattage, comparée ou non avec celle des espèce parentales. Olver et al (1977) ont montré que jusqu'à l'âge de 10 semaines, les canards communs et mulards étaient significativement plus lourds que les canards de Barbarie et les hinnies. Par ailleurs, jusqu'à l'âge de 7 semaines, les hinnies étaient significativement plus lourds que les canards de Barbarie, ce qui correspond au fait que les Barbarie sont des animaux peu précoces. Dans cette étude, cependant, les deux sexes étaient mélangés et l'influence de ce facteur n'était pas étudiée. En comparant des animaux de même sexe, Tai et Rouvier (1998) ont également montré qu'à un âge précoce (4 semaines), chez les mâles, les canards de Barbarie sont les plus légers, les communs les plus lourds, les hinnies étant intermédiaires et les mulards identiques aux communs. A partir de 10 semaines, ce sont les mâles Barbarie qui sont les plus lourds et les mâles communs les plus légers, les deux croisés étant complètement intermédiaires. Larzul et al (2002) ont montré que chez les mâles, dès l'âge de 6 semaines, les canards de Barbarie pouvaient être les plus lourds, les canards communs les plus légers et les deux types croisés toujours intermédiaires. Des différences de classement sur la croissance précoce des types génétiques entre des études s'expliqueront avant tout par le choix des souches de canards communs, plus ou moins lourdes suivant l'étude.

Chez le canard mulard, comme chez le canard Pékin, il n'apparaît pas de dimorphisme sexuel (figure 2), au contraire du canard de Barbarie et du hinny (Tai et Rouvier 1998). Ces résultats mettaient en évidence l'influence de la femelle Barbarie sur le dimorphisme sexuel pondéral, en ralentissant la croissance de sa descendance femelle sans affecter celle de sa descendance mâle, que ce soit en pur ou en croisement. Les auteurs suggéraient qu'audelà des effets habituels des gènes liés au sexe, il existerait des gènes dans la région non pseudo-autosomale du 
Figure 2. Evolution du poids vif des canards mulards mâles et femelles en fonction de l'âge (Baéza et al 2000).

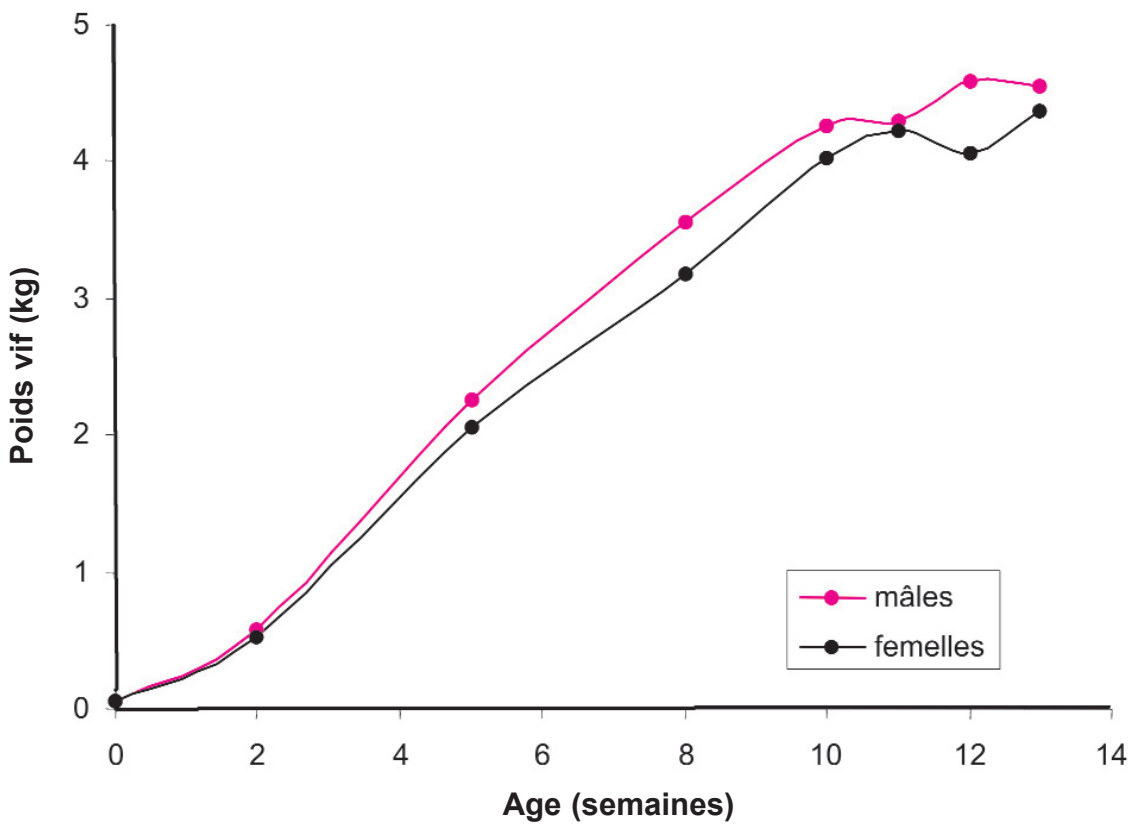

chromosome W du Barbarie pouvant ralentir la croissance par rapport au chromosome W du Pékin. Cette hypothèse n'a pas encore été vérifiée par des méthodes moléculaires.

Les différences de croissance entre les espèces parentales et les deux croisés s'expliquent en termes de paramètres du croisement décrits par Dickerson (1969) en distinguant les effets génétiques additifs directs, maternels et d'hétérosis. Chez le canard mâle, les différences de croissance entre les espèces parentales s'expliquent essentiellement par les effets additifs directs, les effets maternels étant significatifs uniquement en début de croissance, jusqu'à 4 ou 6 semaines d'âge (Tai et Rouvier 1998, tableau 1).
Les effets additifs directs du Barbarie sont d'abord inférieurs à ceux du Pékin à l'âge de 4 semaines, puis deviennent supérieurs à ceux transmis par le mâle Pékin entre les âges de 4 et 6 semaines. En ce qui concerne la supériorité des croisés par rapport aux espèces parentales pour la croissance, les résultats de ces deux études montrent des effets d'hétérosis direct de l'ordre de $4 \%$ chez les mâles en début de croissance. Tai et Rouvier (1998) ont également montré qu'aux âges tardifs, à partir de 16 semaines, les canards mâles croisés ont des performances de croissance inférieures à la moyenne parentale, avec des écarts de l'ordre de -8 à $-12 \%$. Chez les femelles, les effets additifs directs vont dans le même sens que ceux observés chez les mâles, avec cependant des différences entre espèces parentales moins élevées que celles relevées chez les mâles (du même ordre de grandeur entre 0 et 10 semaines et de l'ordre de 30 à $50 \%$ moins élevées entre 16 et 30 semaines d'âge). Les effets maternels sont très significatifs sur la croissance des femelles, les effets maternels de la cane Barbarie étant très défavorables dès l'âge de 4 semaines. Enfin, on n'observe quasiment pas d'effet d'hétérosis chez les canes croisées, contrairement à ce qui est observé chez les mâles croisés.

Pour les paramètres génétiques de la croissance, les héritabilités ont été estimées dans des populations de canard de Barbarie (Mignon-Grasteau et al 1998, $\mathrm{Hu}$ et al 1999) et de Pékin (Pingel 1990b, Brun et Larzul 2003). Les héritabilités du poids vif à 6 semaines chez le mulard en croissance ont été estimées en considérant le poids du mulard comme une mesure répétée attribuée à la cane commune, mère du mulard. Lorsqu'elles sont estimées conjointement, l'héritabilité du poids du mulard est toujours inférieure à celle du poids du canard Pékin : 0,29 vs 0,55 (Brun et Larzul 2003), 0,25 vs 0,37, (Larzul $2002 b)$. De plus, la corrélation génétique entre ces deux poids est élevée $(0,64$ et 0,74$)$. Sélectionner sur le poids du canard Pékin permet donc d'améliorer efficacement le poids du canard mulard.

\section{2 / La couleur du plumage}

Une souche de Pékin (dont la coloration du plumage est blanche), accouplée à une souche blanche de Barbarie peut donner des mulards colorés. Certaines souches de Pékin utilisées

Tableau 1. Moyennes et paramètres du croisement pour les caractères de croissance et de gavage (Imbert 2002).

\begin{tabular}{|c|c|c|c|c|c|c|c|c|}
\hline & $B^{1}$ & $\mathbf{H}$ & M & $\mathbf{P}$ & $\mathrm{ET}^{2}$ & $\begin{array}{c}\text { Effets } \\
\text { additifs } \\
\text { directs } \\
\qquad \mathrm{g}_{\mathrm{B}}^{\prime}{ }^{3}\end{array}$ & Hétérosis ${ }^{3}$ & $\begin{array}{c}\text { Effets } \\
\text { maternels } \\
g_{B}^{M}{ }_{B}^{3}\end{array}$ \\
\hline \multicolumn{9}{|l|}{ Poids corporels (g) } \\
\hline 6 semaines & $2785 a$ & $2470 b$ & $2353 b$ & $1835 c$ & 167 & $417^{*}$ & $101^{*}$ & $59^{*}$ \\
\hline 11 semaines & $4366 a$ & $3527 b$ & $3442 b$ & $2508 c$ & 272 & $886^{*}$ & 48 & 43 \\
\hline 13 semaines (entrée en gavage) & $4837 a$ & $3849 b$ & $3719 b$ & $2645 c$ & 290 & $1032^{*}$ & 43 & 65 \\
\hline 15 semaines (fin de gavage) & $6520 a$ & $5714 b$ & $5774 b$ & $4095 c$ & 292 & $1243^{*}$ & $437^{*}$ & -30 \\
\hline Poids de foie gras $(\mathrm{g})$ & $495 b$ & $493 b$ & $588 a$ & $286 \mathrm{c}$ & 85 & $152^{*}$ & $150^{*}$ & $-48^{*}$ \\
\hline Gain de poids en gavage (g) & $1683 c$ & $1865 b$ & $2052 a$ & $1450 d$ & 189 & $210^{*}$ & $392^{*}$ & $-93^{*}$ \\
\hline
\end{tabular}

${ }^{1} \mathrm{~B}=$ Barbarie, $\mathrm{H}=$ hinny, $\mathrm{M}=$ mulard, $\mathrm{P}=$ Pékin ; ${ }^{2} \mathrm{ET}=$ Ecart-type $;{ }^{3}$ Les effets génétiques additifs directs du Barbarie gl $\mathrm{B}$ sont définis tels que, si

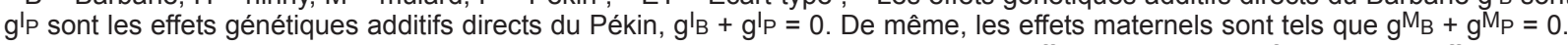

abcd : Pour chaque caractère, les moyennes des moindres carrés avec des lettres différentes sont significativement différentes $(P \leq 0.05)$;

* $: \mathrm{P}<0.05$ pour le seuil de significativité des effets génétiques. 
comme mère du mulard ont été sélectionnées sur la coloration blanche des mulards, afin que les carcasses offrent une meilleure présentation, avec des sicots (repousse de plumes) peu visibles. Ce type de sélection avait déjà été initié à Taiwan dans le milieu des années 1970 (Huang 1985, Lee et Kang 1997). Dans ces études, la sélection des canes communes pour le blanchiment du mulard s'effectue sur descendance en donnant une note reflétant l'extension de la couleur du duvet du caneton éclos, sur une échelle de 1 à 15 . En France, il existe également une sélection de ce type, en notant l'extension de la couleur sur une échelle de 1 à 6 (rappelée par Guémené et al 2004b). Ce caractère a une héritabilité de l'ordre de 0,4 (Huang 1985).

Le blanchiment complet des canards mulards n'est pas systématiquement recherché : l'objectif est en effet de garder une tache de couleur au niveau de la tête (cf. photo) afin de pouvoir réaliser un autosexage sur la couleur du duvet. Le gène chocolat, porté par le chromosome $\mathrm{Z}$, dont l'allèle récessif $\mathrm{ch}$ donne une couleur de plumage marron, permet, lorsque l'allèle chocolat est fixé dans la population Barbarie, de distinguer les mâles mulards $\left(\mathrm{Z}_{\mathrm{ch}} \mathrm{Z}_{\mathrm{Ch}}\right.$ couleur de la tache noire) des femelles mulardes $\left(\mathrm{Z}_{\mathrm{ch}} \mathrm{W}\right.$ couleur de la tache chocolat). Ce gène a été mentionné chez le canard de Barbarie par Hollander (1970) mais il est également présent dans de nombreuses espèces aviaires (Carefoot 1996).

\section{3 / Le comportement}

Les travaux sur le comportement du canard mulard ont surtout porté sur les réactions de stress et d'émotivité face à l'acte de gavage, en réponse à la mise en cause de cette pratique pour le bienêtre animal. Les résultats disponibles montrent que l'acte de gavage n'est pas une source majeure de stress pour le mulard lorsqu'il est en cage individuelle puisqu'il n'y a pas d'augmentation de la corticostéronémie, indicateur de stress aigu, suite à cet acte (Guémené et al 2004a). Au cours de ces expériences, il est cependant apparu que le canard mulard présente une grande sensibilité comportementale, notamment à des actes physiques tels que la capture et la contention, potentiellement sources de stress aigu et/ou chronique. Le problème du comportement du mulard a parallèlement été soulevé avec le blanchiment des souches de canards communs utilisées comme mère du mulard, avec notamment l'apparition de comportements induisant des difficultés d'élevage. Cette hypothèse n'a cependant pas été vérifiée. Les mulards à plumage blanc n'ont pas des réponses comportementales sensiblement différentes des mulards à plumage coloré (Guémené et al 2003, Guémené et al $2004 b$ ). Les réponses observées dans les tests d'open-field ou d'immobilité tonique, suggèrent que le canard mulard est un animal peureux ou émotif, et vraisemblablement très sociable (Guémené et al 2004b).

Des travaux ont été menés sur les réactions de stress et de peur à l'homme, en comparaison des espèces parentales (Faure et al 2003). Le mulard montre, suivant le caractère mesuré, des réactions intermédiaires à celles des deux espèces parentales (niveaux de corticostérone), ou identiques à l'un des deux parents (réponses comportementales), ou encore des réactions extrêmes, notamment pour l'évitement à l'homme. Par ailleurs, il existe également une variabilité génétique chez le canard commun pour les réponses comportementales et la corticostéronémie mesurée chez le mulard, avec des héritabilités de l'ordre de 0,3 pour les déplacements dans le test d'open-field et le niveau de corticostérone après un test d'immobilité tonique ou de contention en filet.

\section{4 / L'efficacité alimentaire}

L'efficacité alimentaire est un critère qui se rapporte plutôt à la phase de croissance pour définir l'aptitude du canard à produire davantage de tissu maigre par rapport à la quantité d'aliment ingéré. L'intérêt principal est de réduire les coûts alimentaires en favorisant la croissance des tissus maigres aux dépens des tissus adipeux. La notion peut cependant être étendue à la phase de gavage en mesurant le poids de foie gras obtenu par rapport à la quantité de maïs ingéré, mais elle se traduit plutôt en terme d'aptitude au gavage.

Il a été montré expérimentalement qu'il est possible de diminuer l'indice de consommation du canard Pékin par sélection $\left(\mathrm{h}^{2}=0,21\right.$ pour Clayton et Powell 1979, Powell 1984, $\mathrm{h}^{2}=0,52$ pour Klemm et al 1994). L'indice de consommation augmente largement avec l'âge. Ainsi, chez la canette mularde, l'indice de consommation passe de 2,12 lorsqu'il est mesuré entre 0 et 42 jours à 4,43 lorsqu'il est mesuré entre 0 et 12 semaines d'âge (Guy et al 1998). En production d'animaux non destinés au gavage, en tenant compte de l'âge d'abattage préconisé pour chaque espèce, le canard Pékin mâle a le meilleur indice de consommation puisque, étant le plus précoce, il est abattu plus jeune. Retailleau (1999) trouve des indices de consommation de 2,5 et 2,9 pour des mâles Pékin et Barbarie mesurés entre 0 et respectivement 49 et 84 jours d'âge. Lorsque les espèces sont mesurées à des âges identiques, les mulards présentent une efficacité alimentaire intermédiaire à celle des espèces parentales (Guy et al 1999, Baéza et al 2005). Il n'existe aucune étude portant sur la variabilité génétique de ce caractère chez le mulard. Il semble cependant possible, en sélectionnant sur un critère d'efficacité alimentaire chez le canard commun (Klemm 1995), de diminuer l'indice de consommation dans la descendance mulard (Larzul et al 2004). Ainsi, une différence de 2,13 points d'indice de consommation chez les canes Pékin, mesuré entre 4 et 7 semaines d'âge, se traduit par une différence de 0,7 point dans leur descendance femelle mularde.

\section{5 / La composition corporelle du canard maigre}

Olver et al (1977) ont mis en évidence que, mesurés au même âge en sexes mélangés, les canards communs étaient significativement plus gras que les canards de Barbarie et les 2 hybrides (figure 3). Depuis, quelques auteurs ont confirmé l'adiposité supérieure du Pékin par rapport au Barbarie, le mulard apparaissant le plus maigre, mesurée par le gras abdominal (Chartrin et al 2004) ou l'ensemble gras abdominal/tissus adipeux souscutanés (Ricard et al 1988, Gorski et al 2000). Les dépôts de gras abdominal, en proportion du poids vif, sont équivalents chez le canard de Barbarie et le canard Pékin (Ricard et al 1988, Hermier et al 2003), l'engraissement supérieur des canards Pékin étant essentiellement dû aux tissus adipeux sous-cutanés. Par ailleurs, au-delà de 10 semaines d'âge, les Pékin présentent les proportions de muscle les plus faibles (Ricard et al 1988, Chartrin et al 2003), traduisant la croissance précoce de ce génotype. Les âges habituels d'abattage des différentes espèces se font d'ailleurs en fonction du développement relatif de leurs tissus maigres et gras, les canards Pékin étant abattus les plus jeunes.

\section{6 / L'aptitude au gavage}

Il existe peu d'animaux pour lesquels le gavage conduise à une stéatose hépa- 
Figure 3. Pourcentage de gras total de la carcasse / poids carcasse (Olver et al 1977).

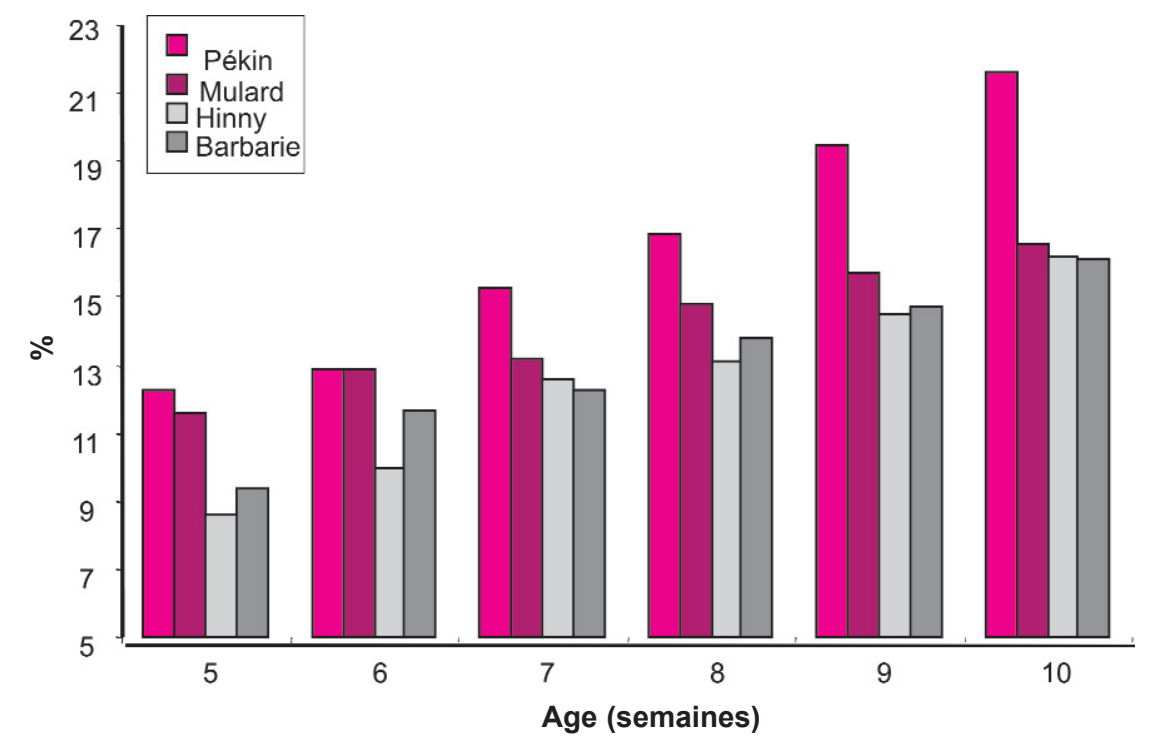

tique spontanée (Hermier et al 2003) et totalement réversible (Babilé et al, Bénard et al 1998). Chez les palmipèdes domestiques tels que l'oie, le canard de Barbarie et surtout le canard mulard, cette capacité est exploitée pour la production de foie gras, c'est-àdire un foie dont le poids est supérieur réglementairement à $300 \mathrm{~g}$ chez le canard et à $400 \mathrm{~g}$ chez l'oie. A l'heure actuelle, ces niveaux de production sont largement dépassés grâce aux améliorations des techniques de gavage et à l'utilisation de souches sélectionnées (Guy et Guémené 2004, Robin et al 2004). Chez l'oie, le gavage peut aboutir à une multiplication du poids du foie par 10 en 2 semaines, pour représenter $10 \%$ du poids de l'animal, par accumulation de lipides, essentiellement des triglycérides, synthétisés de novo à partir de l'amidon contenu dans la ration.

Chez le canard mulard, les résultats observés sont extrêmement variables selon l'étude, mais suivant le type génétique, on a pu montrer des niveaux relatifs de poids de foie de $8,5-9 \%$ du poids vif (Larzul et al 2000, Guy et al 2002). Dans une étude comparant le mulard et les types parentaux, Larzul et al (2002) ont constaté que le pourcentage de poids de foie du mulard (et du hinny) était largement supérieur à celui de ses deux parents Barbarie et canard commun (10,2 et 8,6, 7,5 et $7 \%$ respectivement). Le canard Pékin est d'ailleurs réputé pour ne pas produire de foie gras. Cependant, lorsque le poids de foie est rapporté au poids vif, il peut atteindre en pourcentage des niveaux d'engraissement comparables à celui du Barbarie (Larzul et al 2002, Hermier et al 2003).
Les mécanismes physiologiques d'obtention du foie gras ont été décrits chez les oies (Hermier et al 1991, Fournier et al 1997, Mourot et al 2000). Ils ont également été partiellement décrits chez le canard commun et le canard de Barbarie (Hermier et al 2003) et chez le canard mulard (Guy et al 1999) pour expliquer les différences d'aptitude à l'engraissement des différents génotypes. Cet aspect a fait récemment l'objet d'une revue bibliographique (Baéza et al 2005). D'après ces auteurs, le Barbarie a une lipogénèse hépatique plus efficace et accumule davantage les triglycérides dans le foie. Il est le plus efficace pour transformer le maïs en foie gras. A l'opposé, le canard Pékin se caractérise surtout par une plus forte exportation des acides gras produits par le foie vers les tissus adipeux périphériques. Les canards mulards et hinnies ont des niveaux d'engraissement périphérique intermédiaires. Les mécanismes d'accumulation des lipides dans le foie chez le mulard sont donc comparables à ceux observés dans les espèces parentales, l'aptitude au gavage de ces animaux étant surtout liée à leur capacité d'ingestion. Lorsqu'ils sont gavés selon leur capacité d'ingestion (Larzul et al 2002), les canards mulards et hinnies ingèrent une quantité supérieure de maïs par rapport aux Barbarie $(10 \mathrm{~kg} v s$ $8,1 \mathrm{~kg}$ ) pour un poids vif d'entrée en gavage inférieur de plus de $1 \mathrm{~kg}(3,7 \mathrm{~kg}$ vs 4,8 kg). Dans cette même étude, les canards Pékin consommaient également $8 \mathrm{~kg}$ de maiis pour un poids vif d'entrée en gavage de 2,6 kg. Dans le cadre du croisement factoriel entre une souche de Barbarie et une souche de Pékin, le poids du foie gras bénéficie d'un fort effet d'hétérosis, des effets génétiques additifs directs favorables du mâle Barbarie et des effets maternels favorables de la cane Pékin. Le gain de poids en gavage est soumis aux mêmes effets (tableau 1). Le mulard en gavage bénéficie donc d'une complémentarité entre les effets additifs directs et maternels favorables des deux parents, ce qui explique une aptitude au gavage supérieure à celle du meilleur parent. Au niveau des populations de canes communes utilisées comme mères du mulard, des estimations de paramètres de croisement ont été réalisées dans le croisement d'une population de Pékin lourd et d'une population de Tsaiya (Rouvier et al 1994). Ces auteurs ont trouvé que les différences des effets génétiques additifs directs sur les caractères de croissance et foie gras des mulards étaient significatives en faveur de la souche Pékin, sauf sur le taux de fonte (pertes lipidiques au cours de la cuisson). L'hétérosis maternel était en général significatif et favorable, de $6,2 \%$ pour le poids de foie et de $-2,8 \%$ pour le taux de fonte lipidique du foie à la stérilisation. Ces résultats indiquent que le croisement entre un mâle Pékin lourd et une cane commune légère très féconde serait à considérer pour améliorer la production de foie gras d'un mulard léger. La connaissance de l'influence du format de la cane Pékin sur les performances de croissance et gavage de ses produits mulards permet d'orienter rapidement les croisements dans le sens des objectifs du marché sur le poids des produits (foie gras, paletots, magrets). Larzul et al (1999) ont montré que pour une même souche de mâles Barbarie, le poids corporel de la souche de mère du mulard a surtout une influence sur la croissance et le développement musculaire et dans une moindre mesure sur le poids du foie de ses mulards.

La sélection directe dans une population de Barbarie pour le poids du foie, avec des mesures effectuées sur canards de Barbarie gavés, est efficace avec des valeurs estimées de l'héritabilité variant de 0,20 à 0,40 (Babilé 1989, résultats rapportés par Poujardieu et al 1994, Mignon-Grasteau et al 1998). Si cette sélection est susceptible d'améliorer également les performances du canard mulard, aucun résultat chiffré publié n'existe pour étayer ce point. Trois études ont porté sur l'estimation de l'héritabilité, dans des populations de canards communs, de l'aptitude au gavage mesurée sur des mulards. L'héritabilité varie entre 0,09 (Larzul, 2002a,b) et 0,17 (Poujardieu et al 1994). Toutefois, les souches étudiées, 
les conditions d'élevage et de gavage, ainsi que les modèles statistiques ne sont pas identiques dans ces études et peuvent expliquer des valeurs différentes. Il apparaît cependant clairement que les lignées de canards communs peuvent être sélectionnées sur l'aptitude au foie gras de leurs descendants mulards. Le poids de foie est peu lié au poids en début de gavage $\left(-0,14<r_{g}\right.$ $<0)$. Les poids de paletot et de magret ont des héritabilités plus élevées que le poids du foie $(0,22$ à 0,29$)$ et sont génétiquement très liés au poids vif mesuré en début de gavage $\left(\mathrm{r}_{\mathrm{g}}>0,85\right)$. L'analyse des corrélations génétiques montre qu'en ne sélectionnant que sur un poids corporel, que ce soit celui des canards communs ou celui des mulards, l'évolution du poids du foie est très limitée voire nulle.

\section{7 / La qualité des produits}

Le principal critère de qualité du foie gras est le taux de fonte, c'est-à-dire les pertes lipidiques au cours de la cuisson. Le canard mulard, qui présente un meilleur rendement de foie par rapport au poids vif, ou par rapport au poids de carcasse, présente également des foies de meilleure qualité en terme de fonte. En moyenne, le taux de fonte mesuré sur un échantillon de $60 \mathrm{~g}$ en condition de stérilisation, est supérieur de 10 points chez le Barbarie par rapport au mulard (45 vs $35 \%$ pour Babilé et al
1988, 55,6 \% vs 43,9 \% pour Salichon et al 1993). S'il est indéniable que plus les foies sont lourds, plus ils sont fondants, les paramètres biochimiques qui permettraient de prédire la qualité du foie sont encore inconnus, même s'il existe quelques résultats intéressants chez l'oie concernant l'influence de la composition et de la fluidité des membranes des hépatocytes (BouillierOudot et al 2000). Quelques études ont également été réalisées sur d'autres critères de qualité tels que la couleur, la texture ou les qualités organoleptiques, mais essentiellement pour comparer des canards mulards issus de différentes souches. Il existe ainsi une grande variabilité de la qualité organoleptique $\mathrm{du}$ foie gras suivant les génotypes parentaux utilisés pour produire le canard mulard (Robin et al 2002), sans que le déterminisme génétique de ces différences ait été analysé.

Les caractéristiques de la viande de canard ont fait l'objet de plusieurs revues bibliographiques (Baéza 1995, Baéza et al 2005), que ce soit pour des animaux gavés ou non gavés. Les différences de composition du muscle sont significatives entre les canards Pékin et les canards de Barbarie, les caractéristiques des canards hybrides apparaissant intermédiaires pour les critères de $\mathrm{pH}$, de couleur du muscle, de texture ou de composition chimique. Les principales différences se retrouvent au niveau de la teneur en lipides intramusculaires, de la composition en acides gras et de la texture de la viande. Imbert (2002) a estimé les paramètres du croisement pour les mesures de qualité de viande. Les effets d'hétérosis et les effets maternels sont peu significatifs sur les caractéristiques physico-chimiques, chimiques, et de texture de la viande. Les différences entre types génétiques, lorsqu'elles sont significatives, sont essentiellement dues aux effets additifs directs (tableau 2) avec surtout des effets défavorables du Barbarie sur la texture de la viande. Pour les autres critères, il est difficile de juger si les effets du Barbarie sont favorables ou défavorables.

Pour la qualité du foie, Poujardieu et al (1994) ont estimé l'héritabilité de la perte d'exsudat lipidique à la stérilisation à 0,18 , avec une corrélation génétique très élevée avec le poids du foie $(0,89)$. A notre connaissance, aucune estimation d'héritabilité n'a été réalisée chez le canard pour la qualité sensorielle de la viande.

\section{4 / Eléments de génétique moléculaire}

Hormis l'étude du fonctionnement spécifique du génome du canard mulard où s'expriment de manière

Tableau 2. Moyennes et paramètres du croisement pour les caractères de composition chimique du magret (Imbert 2002).

\begin{tabular}{|c|c|c|c|c|c|c|c|c|}
\hline & $\mathbf{B}^{1}$ & $\mathbf{H}$ & $\mathbf{M}$ & $\mathbf{P}$ & $E T^{2}$ & $\begin{array}{c}\text { Effets } \\
\text { additifs } \\
\text { directs } \\
\qquad \mathrm{g}_{\mathrm{B}}^{\prime}{ }^{3}\end{array}$ & Hétérosis ${ }^{3}$ & $\begin{array}{c}\text { Effets } \\
\text { maternels } \\
\mathrm{g}_{\mathrm{B}}^{\mathrm{M}^{3}}\end{array}$ \\
\hline $\mathrm{pH} 24 \mathrm{~h}$ & 5,79 & 5,84 & 5,80 & 5,81 & 0,06 & $-0,03^{*}$ & 0,02 & 0,02 \\
\hline$L^{*}$ & $46,0 a$ & $42,9 b$ & $44,2 a b$ & $44,9 a b$ & 2,3 & $1,17^{*}$ & $-1,92^{*}$ & $-0,66$ \\
\hline$a^{*}$ & 21,1 & 21,2 & 21,5 & 21,4 & 1,1 & $-0,03$ & 0,07 & $-0,12$ \\
\hline$b^{*}$ & 8,97 & 8,14 & 9,05 & 9,28 & 1,5 & 0,30 & $-0,53$ & $-0,46$ \\
\hline Pertes à la cuisson (\%) & $24,57 a$ & $21,09 b$ & $19,43 b$ & $20,63 b$ & 3,0 & 1,1 & $-2,3^{*}$ & 0,8 \\
\hline Extrait sec (\%) & 28,29 & 27,51 & 27,82 & 28,07 & 1,42 & 0,27 & $-0,51$ & $-0,16$ \\
\hline Minéraux (\%) & 1,23 & 1,20 & 1,16 & 1,15 & 0,08 & 0,01 & $-0,01$ & 0,02 \\
\hline Lipides (\%) & $4,91 b$ & $5,34 a b$ & $5,81 a b$ & $6,30 a$ & 1,03 & $-0,42$ & 0 & $-0,27$ \\
\hline Protéines (\%) & $22,93 a$ & $21,96 b$ & $21,68 b$ & $21,31 b$ & 0,86 & $0,67^{*}$ & $-0,31$ & 0,14 \\
\hline Solubilité du collagène (\%) & $12,99 b$ & $12,77 b$ & $14,27 \mathrm{ab}$ & $18,86 a$ & 3,6 & $-2,2^{*}$ & $-2,4^{*}$ & $-0,8$ \\
\hline Collagène total & $4,82 a$ & $4,38 a b$ & $4,45 a$ & $4,30 \mathrm{~b}$ & 0,60 & $0,30^{*}$ & $-0,15$ & $-0,04$ \\
\hline Collagène thermosoluble & $0,632 b$ & $0,548 b$ & $0,637 a b$ & $0,815 a$ & 0,20 & $-0,05$ & $-0,13^{*}$ & $-0,04$ \\
\hline \multicolumn{9}{|l|}{ Warner-Bratzler test } \\
\hline Force max & $62,6 a$ & $46,0 \mathrm{ab}$ & $46,6 a b$ & $33,2 b$ & 9,6 & $15,0^{*}$ & $-1,6$ & $-0,3$ \\
\hline Energie au max & $220 a$ & $160 a b$ & $160 a b$ & $107 b$ & 41 & $57^{*}$ & -4 & 0 \\
\hline
\end{tabular}

${ }^{1} \mathrm{~B}=$ Barbarie, $\mathrm{H}=$ hinny, $\mathrm{M}=$ mulard, $\mathrm{P}=$ Pékin $;{ }^{2} \mathrm{ET}=$ Ecart-type $;{ }^{3}$ Les effets génétiques additifs directs du Barbarie gl $\mathrm{B}$ sont définis tels que, si $g^{l} \mathrm{P}$ sont les effets génétiques additifs directs du Pékin, $g^{l_{B}}+g^{l_{P}}=0$. De même, les effets maternels sont tels que $g^{M_{B}}+g^{M_{P}}=0$. abc : Pour chaque caractère, les moyennes des moindres carrés avec des lettres différentes sont significativement différentes ( $\mathrm{P} \leq 0.05)$;

* $: \mathrm{P}<0.05$ pour le seuil de significativité des effets génétiques. 
conjointe les génomes du canard commun et du canard de Barbarie, notamment pour expliquer la faible fertilité dans l'accouplement mâle Barbarie $\mathrm{x}$ cane commune (Tchelycheva et al 1993), les connaissances en génétique moléculaire sont développées dans les espèces parentales et s'appliquent très directement au génome du canard mulard.

\section{1 / Marqueurs}

Les premiers marqueurs microsatellites ont été développés pour des études de diversité génétique soit chez le canard de Barbarie (Stai et Hughes 2003) soit chez le canard commun (Fields et al 1997, Takahashi et al 2001, Maak et al 2003) ou chez ce dernier pour des études de parenté (Denk et al 2004). Le polymorphisme semble plus élevé dans les populations sauvages que dans les populations domestiques. C'est clairement démontré dans le cas des canards de Barbarie avec un nombre moyen d'allèles de 11 et de 3,5, respectivement pour les variants sauvages et domestiques (Stai et Hughes 2003) et seulement suggéré dans les populations de canard commun (Maak et al 2003). Les premières cartes génétiques sont en cours de réalisation pour le canard commun avec des marqueurs AFLP (Taiwan) ou microsatellites (Chine, Huang et al 2004, 2005) et pour le canard commun et le canard de Barbarie avec des marqueurs microsatellites (France). La mise en commun de ces données devrait aboutir à l'obtention de quelques centaines de marqueurs polymorphes utilisables tant chez le canard commun que chez le canard de Barbarie et donc sur le mulard.

\section{2 / Cartographie comparée}

Les travaux de cartographie comparée ont montré la grande conservation des génomes aviaires (Shetty et al 1999, Fillon et al 2001, Guttenbach et al 2003). De plus, les sondes de microchromosomes de poule ayant donné des signaux d'hybridation chez le canard sont également localisées sur les micro- chromosomes de canard (Fillon et al 2001). Quelques réarrangements ont cependant été observés pour le génome du canard par rapport à celui du poulet sur la plupart des macrochromosomes exceptés pour les macrochromosomes 1 et 2 (Fillon et al 2001).

\section{Conclusion}

$\mathrm{Au}$ cours de cet article, nous avons effectué une revue des connaissances concernant le déterminisme génétique d'un certain nombre de caractères du mulard. Les deux espèces parentales (le canard commun et le canard de Barbarie) sont génétiquement isolées depuis 50 millions d'années et présentent de nombreuses spécificités biologiques (durée d'incubation, modes de vie aquatique ou non, comportement, etc.) et zootechniques (précocité de croissance, aptitude à la stéatose hépatique, engraissement de la carcasse et de la viande). Cependant, ces deux espèces sont inter-fertiles, avec un taux de fertilité moyen des canes communes inséminées avec de la semence de Barbarie qui peut atteindre $80 \%$. La différence d'espèce se traduit par une durée de fertilité moins grande en croisement, un léger surcroît de mortalité embryonnaire précoce des embryons mulards par rapport aux embryons «communs» et surtout la stérilité des mulards. L'amélioration par sélection doit donc passer par une sélection dans les souches parentales. En France, le mulard est actuellement exploité dans le cadre du gavage pour la production de foie gras et de viande. Pour de nombreux caractères zootechniques, le mulard est intermédiaire entre les souches parentales, ce qui laisse supposer un déterminisme génétique «additif» de ces caractères chez le mulard. On peut alors s'attendre à ce qu'une amélioration en soi des populations parentales se traduise au niveau du mulard. Ceci se vérifie expérimentalement pour la croissance et l'efficacité alimentaire, par exemple. Cela semble plus hypothétique pour d'autres caractères pour lesquels le mulard n'est pas intermé- diaire entre les espèces parentales comme l'aptitude à la stéatose hépatique ou encore les caractères de comportement. Pour ces caractères, la sélection des parents doit se faire directement sur les performances du mulard à moins que la corrélation génétique entre la valeur d'un géniteur en souche pure et sa valeur en hybridation soit élevée. Les perspectives d'amélioration génétique du mulard reposent sur une connaissance accrue du déterminisme génétique de ses caractères. Pour la filière foie gras, les enjeux résident dans une meilleure adaptation des génotypes aux nouvelles normes de gavage qui se mettront progressivement en place, avec notamment une amélioration de la facilité de gavage. En génétique quantitative classique cela passe une meilleure connaissance des héritabilités et corrélations génétiques entre caractères, mais aussi par le développement de modèles statistiques de l'hérédité, prenant en compte simultanément les deux parents du mulard. La génétique moléculaire offre des perspectives intéressantes du point de vue de la sélection puisque, pour la production des canards mulards, il s'agit souvent de caractères peu à moyennement héritables, pour des performances qui ne sont pas mesurables directement dans les espèces parentales. D'un point de vue plus académique, la génétique moléculaire pourrait apporter des réponses par rapport à la compatibilité génétique entre les deux espèces parentales et à la stérilité des hybrides.

\section{Remerciements}

Une grande partie des travaux cités ont été réalisés à l'Unité Expérimentale sur les Palmipèdes à Foie Gras (INRA Artiguères, Landes) sous la direction de Daniel Rousselot-Pailley puis de Gérard Guy. A ce titre, les auteurs remercient l'ensemble du personnel de cette station. Leurs remerciements vont également à l'interprofession (CIFOG) pour son soutien financier dans de nombreux programmes.

\section{Références}

Babilé R., 1989. La production de foie gras de canards de Barbarie (Cairina moschata) : aspects génétiques, nutritionnels et technologiques, Thèse de Doctorat, Institut polytechnique de Toulouse, $315 \mathrm{p}$.

Babilé R., Auvergne A., Dubois J.P., Bénard G.,
Manse H., 1998. Réversibilité de la stéatose hépatique chez l'oie. $3^{\mathrm{e}}$ Journ. Rech. Palmipèdes à Foie Gras, Bordeaux, France, 27-28 Oct. 1998, 45-48.

Baéza E., 1995. La viande de canard : production et principales caractéristiques. INRA Prod. Anim., 8, 117-125.
Baéza E., de Carville H., Salichon M.R., Marché G., Leclercq B., 1997. Effects of selection over three and four generations on meat yield and fatness in Muscovy ducks. Br. Poult. Sci., 38, 359-365.

Baéza E., Salichon M.R., Marché G., 
Wacrenier X., Dominguez B., Culioli J., 2000. Effects of age and sex on the structural, chemical and technological characteristics of mule duck meat. Br. Poult. Sci., 41, 300-307.

Baéza E., Rideau N., Chartrin P., Davail S., Hoo-Paris R., Mourot J., Guy G., Bernadet M.D., Hermier D., 2005. Canards de Barbarie, Pékin et leurs croisements : aptitude à l'engraissement. INRA Prod. Anim., 18 (2), 131-141.

Batellier F., Marchal F., Scheller M.F., Gautron J., Sellier N., Taouis M., Monbrun C., Vignal A., Brillard J.P., 2004. Sex-ratios in mule duck embryos at various stages of incubation. Theriogenology, 61, 573-580.

Bebak, 1959. The histological background of fertility in an intergeneric cross. Bull. Acad. Pol. Sci., ser. Sci. Biol., 7, 9, 371-375.

Bénard G., Bénard P., Prehn D., Bengone T., Jouglar J.Y., Durand S., 1998. Démonstration de la réversibilité de la stéatose hépatique obtenue par gavage de canards mulards. Etude réalisée sur 3 cycles de gavage-dégavage. $3^{\mathrm{e}}$ Journ. Rech. Palmipèdes à Foie Gras, Bordeaux, France, 2728 Oct. 1998, 49-52.

Bouillier-Oudot M., Cazeils J.L., Babilé R., Auvergne A., 2000. Relation entre la composition de la membrane plasmique des hépatocytes de foies gras d'oies et leurs caractéristiques technologiques et organoleptiques. $4^{\mathrm{e}}$ Journ. Rech. Palmipèdes à Foie Gras, Arcachon, France, 4-5 Oct. 2000, 139-142.

Brillard J.P.,1993. Sperm storage and transport following natural mating and artificial insemination. Poult. Sci., 72, 923-928.

Brody S., 1945. Bioenergetics and growth. Reinhold Publ. Corp., New York, 484-494.

Brun J.M., Sellier N., Hu Y.H., Rouvier R., 1999. Fertility and embryonic mortality in the intergeneric cross between the common duck and the Muscovy duck: candling vs. opening the eggs. Proc. $1^{\text {st }}$ World Conf. Waterfowl, Taichung, Taiwan, 1-4 Déc. 1999, 193-198.

Brun J.M., Richard M.M., Sellier N., Brillard J.P., 2002. Génétique de la durée de période fertile de la cane commune INRA44 inséminée avec de la semence de canards communs ou de Barbarie : $2^{\mathrm{e}}$ estimation. $5^{\mathrm{e}}$ Journ. Rech. Palmipèdes à Foie Gras, Pau, France, 9-10 Oct. 2002, 21-24.

Brun J.M., Larzul C., 2003. Inheritance of reproductive traits of female common ducks (Anas platyrhynchos) in pure breeding and in inter-generic crossbreeding with Muscovy ducks (Cairina moschata). Br. Poult. Sci., 44, 1-6.

Brun J.M., Sellier N., Richard M.M., Batellier F. Brillard J.P., 2004. Effects of sperm storage duration and age of the common duck (Anas platyrhynchos) on fertility and embryonic survival in pure- or cross-breeding with muscovy drakes (Cairina moschata). XXII ${ }^{\text {nd }}$ World Poult. Congr., Istanbul, Turquie, June 8-13, 2004, comm. O2.

Carefoot W.C., 1996. Chocolate: a sex-linked recessive plumage colour mutant of the domestic fowl. Br. Poult. Sci., 37, 867-868.

Chen B.J., 1992. Nutrient requirements of laying Brown Tsaiya ducks. $9^{\text {th }}$ Int. Symp. Waterfowl, Pise, Italie, 146.

Cheng Y.S., Rouvier R., Poivey J.P., Tai C., 1995. Genetic parameters of body weight, egg production and shell quality traits in the Brown Tsaiya laying ducks. Genet. Sel. Evol., 27, 459472 .
Cheng Y.S., Rouvier R., Poivey J.P., Tai J.J.L., Tai C., Huang S.C., 2002. Selection response for the number of fertile eggs of the Brown Tsaiya duck (Anas platyrhynchos) after a single insemination with pooled Muscovy (Cairina moschata) semen. Genet. Sel. Evol., 34, 597-611.

Chartrin P., Schiavone A., Bernadet M.D., Guy G., Mourot J., Duclos M.J., Baéza E., 2004. Effet du génotype et du gavage sur les dépôts de lipides intramusculaires dans le filet de canard. $6^{\mathrm{e}}$ Journ. Rech. Palmipèdes à Foie Gras, Arcachon, France, 7-8 Oct. 2004, 49-52.

Clayton G.A., Powell J.C., 1979. Growth, food conversion, carcass yields and their heritabilities in ducks (Anas platyrhynchos). Br. Poult. Sci., 20, 121-127.

Denjean B., Ducos A., Darre A., Pinton A. , Seguela A., Berland H., Blanc M. F. , Fillon V., Darré R., 1997. Caryotypes des canards commun (Anas platyrhynchos), Barbarie (Cairina moschata) et de leur hybride. Rev. Méd. Vét., 148, 695-704.

Denk A.G., Gautschi B., Carter K., Kempenaers B., 2004. Seven polymorphic microsatellite loci for paternity assessment in the mallard (Anas platyrhynchos). Mol. Ecol. Notes, 4, 506-508.

Dickerson G., 1969. Experimental approaches in utilizing breed resources, Anim. Breed. Abstr., 37, 191-202.

Duhart F., 2004. Pour une ethnozoologie historique des palmipèdes en Europe : la naissance du foie gras moderne (XVIe- XIX ${ }^{\mathrm{e}} \mathrm{S}$.). $6^{\mathrm{e}}$ Journ. Rech. Palmipèdes à Foie Gras, Arcachon, France, 7-8 Oct. 2004, 9-12.

Etches R.J., 1996. Reproduction in poultry. CAB International, $318 \mathrm{p}$.

Faure, J.M., Val-Laillet, D., Guy, G., Bernadet, M-D , Guémené, D., 2003. Fear and stress reactions in two species of duck and their hybrid. Horm. Behav., 43, 568-572.

Fields R. L., Scribner K.T., 1997. Isolation and characterization of novel waterfowl microsatellite loci: cross-species comparisons and research applications. Mol. Ecol., 6, 199-202.

Fillon V., Vignoles M., Garrigues A., Crooijmans R.P., Groenen M.A., Séguéla A., Gellin J., Vignal A., 2001. Comparative avian cytogenetics: establishment of chromosome correspondence by fluorescent in situ hybridisation. Br. Poult. Sci., 42, 23-24.

Fournier E., Peresson R., Guy G., Hermier D., 1997. Relationships between storage and secretion of hepatic lipids in two breeds of geese with different susceptibility to liver steatosis. Poult. Sci., 79, 599-607.

Gille U., Salomon F.V., 1994. Heart and body growth in ducks. Growth Dev. Aging, 58, 75-81.

Gomot L., 1973. Intersexuality in birds. Study of the effects of hybridization and post-embryonic ovariectomy, special print.

Gomot L., Bonin 1965. L'hybridation canard pékin $\mathrm{x}$ cane de Barbarie. Etude comparée des canards mâles Pékin, Barbarie et hybride. Annales Scientifiques de l'Université de Besançon, 3, 19-38.

Gorski J., Witak B., Biessiada-Drzazga B., Pietkiewicz M., 2000. Comparison between growth of muscular tissue, fatty tissue and liver weight of Pekin ducks, muscovy ducks and crossbred ducks. $21^{\text {st }}$ World Poultry Congress, Montréal, Canada, 20-24 Août 2000.

Guémené D., Fournel E., Faure J.M. , Guy G.,
2003. Effets génotype, phénotype, âge et mode d'élevage sur les réponses comportementales et endocriniennes de canards mulards mâles. In L'éthologie appliquée aujourd'hui, C. Baudoin (ed), Editions ED, Paris, France, 1, 65-72.

Guémené D., Guy G., Faure J.M., 2004a. Foie gras, gavage et bien-être animal : vers un peu d'objectivité ! Le point sur l'évolution des pratiques de production et les acquis de la recherche. $6^{\mathrm{e}}$ Journ. Rech. Palmipèdes à Foie Gras, Arcachon, France, 7-8 Oct. 2004, 81-87.

Guémené D., Larzul C., Bouy S., Bernadet M.D., Guy G., Faure J.M., 2004b. Couleur du plumage et déterminisme génétique des comportements de peur chez le canard mulard. 6e Journ. Rech. Palmipèdes à Foie Gras, Arcachon, France, 7-8 Oct. 2004, 99-104.

Guttenbach M., Nanda I., Feichtinger W., Masabanda J.S., Griffin D.K., Schmid M., 2003 Comparative chromosome painting of chicken autosomal paints 1-9 in nine different bird species. Cytogen. Genome Res., 103, 173-184.

Guy G., Guémené D., 2004. Gavage et production de foie gras. Rétrospectives et perspectives. $6^{\mathrm{e}}$ Journ. Rech. Palmipèdes à Foie Gras, Arcachon, France, 7-8 Oct. 2004, 1-8.

Guy G., Rousselot-Pailley D., Gourichon D., 1995. Comparaison des performances de l'oie, du canard mulard et du canard de Barbarie soumis au gavage. Ann. Zootech., 44, 297-305.

Guy G., Rousselot-Pailley D., Rouvier R., 1998. Description de la croissance de canettes mulardes. Ann. Zootech., 45, 303-310.

Guy G., Hermier D., Davail S., Bely M., Ander J.P., Hoo-Paris R., 1999. Meat production and force-feeding ability of different type of ducks. $1^{\text {st }}$ World Waterfowl Conference, Taichung, Taïwan, 1-4 Dec. 1999, 462-468.

Guy G., Larzul C., Bernadet M.D., 2002 Efficacité alimentaire chez le mulard. 5 e Journ. Rech. Palmipèdes à Foie Gras, Pau, France, 9-10 Oct. 2002, 37-40.

Gvaryahu G., Robinzon B., Meltzer A., Perek M., Snapir N., 1984. Artificial insemination and natural mating in the crossbreeding of the Muscovy drake and the Pekin duck. Poult. Sci., $63,386-387$.

Haldane J.E.S., 1922. Sex ratio and unisexual sterility in hybrid animals. J. Genet., 12, 101109.

Hermier D., Saadoun A., Salichon M.R., Sellier N., Rousselot-Pailley D., Chapman M.J. 1991. Plasma lipoproteins and liver lipids in two breeds of geese with different susceptibility to hepatic steatosis: changes induced by development and force-feeding. Lipids, 26, 331-339.

Hermier D., Guy G., Guillaumin S., Davail S., André J.M., Hoo-Paris R. 2003. Differential channelling of liver lipids in relation to susceptibility to hepatic steatosis in two species of ducks. Comp. Biochem. Physiol. B, Biochem. Mol. Biol., 135, 663-675.

Hetzel D.J.S., 1983. The egg production of intensively managed Alabio and Tegal ducks and their reciprocal crosses. World Rev. Anim. Prod., 19,4 .

Hetzel D.J.S., 1984. Comparative performance of intensively managed Khaki Campbel and Native Indonesian ducks. Trop. Anim. Health Prod, 16, 39-85.

Hollander W.F., 1970. Sex-linked chocolate coloration in the Muscovy duck. Poult. Sci., 49 594-596. 
Hoffman E., 1992. Hybrid progeny from muscovy and domestic ducks. 9 $9^{\text {th }}$ Int. Symp. Waterfowl, Pise, Italie, 64-66.

Hu YH., 1999. Variabilité génétique des performances de croissance et de ponte dans une lignée de canards de Barbarie sélectionnée à Taïwan; Viabilité embryonnaire précoce dans le croisement intergénérique des canards. Thèse de doctorat, Institut National Polytechnique, Toulouse, $125 \mathrm{p}$.

Hu Y.H., Poivey J.P., Rouvier R., Wang C.T., Tai C. 1999. Heritabilities and genetic correlations of body weights and feather length in growing Muscovy selected in Taïwan. Br. Poult. Sci., 40, 605-612.

Huang H.H., 1985. Selection of white mule ducks. J. Chin. Soc. Anim. Sci., 14, 111-122.

Huang H.H., Chow T.C., 1974. Artificial insemination in mule duck production. Proc. XV World. Poult. Congr., New Orleans, USA, 261262.

Huang M.C., Sung Y.Y., 1988. Cytogenetical analysis of the germ cell in the domestic drake and mule drake. Proc. Natl. Sci. Counc, B. ROC, 12 (4), 202-208.

Huang Y., Li N., Hu S., Hao J., Tu J., Zhao L., Hu X., Liu Z., Feng J., 2004. Characterization of 138 novel microsatellite DNA markers and preliminary linkage map for duck (Anas platyrhynchos). 22nd World Poult. Congr., Istanbul, Turquie, June 8-13 2004, Comm. O2.

Huang Y., Tu J., Cheng X., Tang B., Hu X., Liu Z., Feng J., Lou Y., Lin L., Zhao Y., Li N., 2005. Characterization of 35 novel microsatellite DNA markers from the duck (Anas platyrhynchos) genome and cross-amplification in other birds. Genet. Sel. Evol., 37, 455-472.

Imbert B., 2002. Comparaison génétique des caractéristiques biochimiques du magret de canard. Diplôme de Recherche Universitaire de l'Institut National Polytechnique de Toulouse, $63 \mathrm{p}$.

Klemm R., 1995. Selection on feed conversion ratio in ducks. $10^{\text {th }}$ Eur. Symp. Waterfowl, Halle, Allemagne, 26-31 mars 1995, 433-438.

Klemm R., Reiter K., Pingel H., 1994. Results and effects of direct selection for feed efficiency in the domestic ducks: 2. Report: Methodical aspects. Arch. Geflügelk., 58, 176-181.

Knizetova H., Hyanek J., Knize B., Prochatzova H., 1991. Analysis of growth curve of fowl. II. Ducks. Br. Poult. Sci., 22, $1027-$ 1038 .

Knizetova H., Hyanek J., Hyankova L., Belicek P., 1995. Comparative study of growth curves in poultry. Genet. Sel. Evol., 27, 365-375.

Lake P.E., 1975. Gamete production and the fertile period with particular reference to domesticated birds. Symp. Zool. Soc. Lond., 35, $225-$ 244.

Larzul C., 2002a. Genetic parameters for overfed mule duck traits. 7th World Congr. Genet. Appl. Livest. Prod., Montpellier, France, August 19-23 2002, Comm. 04-08.

Larzul C., 2002b. Paramètres génétiques du gavage dans une population de canards communs. 5e Journ. Rech. Palmipèdes à Foie Gras, Pau, France, 9-10 Oct. 2002, 33-36.

Larzul C., Rouvier R., Guy G., RousselotPaillet D., 1999. Influence of female Pekin body size on overfed mule ducks performances. Proc. $1^{\text {st }}$ World Conf. Waterfowl, Taichung, Taiwan, 14 Déc. 1999, 122-126.
Larzul C., Rouvier R., Rousselot-Pailley D., Guy G., 2000. Estimation of genetic parameters in a white plumage "polish" geese strain for growth and carcass traits in non overfed animals, and for growth and overfeeding traits in overfed animals. Genet. Sel. Evol., 32, 415-427.

Larzul C., Imbert B., Bernadet M.D., Guy G., Remignon H., 2002. Qualité du magret dans un croisement factoriel Barbarie x INRA44. $5^{\mathrm{e}}$ Journ. Rech. Palmipèdes à Foie Gras, Pau, France, 9-10 Oct. 2002, 29-32.

Larzul C., Guy G., Bernadet M.D., 2004. Feed efficiency, growth and carcass traits in female mule ducks. Arch. Gefügelk., 6, 265-268.

Lee S.R., Kang C.L., 1997. Improving the percentage of white plumage in Mule ducks. J. Taiwan Livest. Res., 30, 293-299.

Leclercq B., 1990. Croissance et composition corporelle du Canard de Barbarie. In : B. Sauveur et H. de Carville (eds), Le Canard de Barbarie, INRA, Paris, 23-39.

Lutz-Ostertag Y., 1965. Le croisement canard Khaki-Campbell x Cane Barbarie. Arch. Anat. Hist. Embryol., 48, 300-327.

Maak S., Wimmers K., Weigend S., Neumann K., 2003. Isolation and characterization of 18 microsatellites in the Peking duck (Anasplatyrhynchos) and their application in other waterfowl species. Mol. Ecol. Notes, 3, 224-227.

Marchand C.R., Gomot L., 1972. Variations annuelles de l'activité testiculaire du canard hybride issu du croisement Pékin male (Anas $p$ ) $\mathrm{x}$ Femelle Barbarie (Cairina $m$ ). C.R. séances Soc. Biol., 166, 556-561.

Maruyama K., Akbar M.K., Turk C.M., 1999. Growth patterns and carcass development in male ducks selected for growth rate. Br. Poult. Sci., 40, 233-239.

Mialon M.M., 1985. Croisement entre 3 souches de Barbarie et de canes communes : performances de reproduction, de gavage des mulards obtenus. Mémoire de fin d'études, ENITA Bordeaux, 53p.

Mignon-Grasteau S., Beaumont C., Poivey J.P., Rochambeau H. de., 1998. Estimation of the genetic parameters of sexual dimorphism of body weight in "label" chickens and Muscovy ducks. Genet. Sel. Evol., 30, 481-491.

Migliore L., Tesoro M., Romboli I., 1986. Chromosome complement and $\mathrm{C}$ banding pattern in the Muscovy duck. $7^{\mathrm{e}}$ Conf. Eur. Aviculture, Paris, France, 1, 99-103.

Migliore L., Romboli I., Fedeli-Avanzi C., 1988. Génétique du plumage du canard de Barbarie (Cairina Moschata). Une mise au point. Les Colloques de l'INRA, 42, 29-38.

Mott C.L., Lockhart L.H., Rigdon R.H., 1968. Chromosomes of the sterile hybrid duck. Cytogenetics, 7, 403-412.

Mourot J., Guy G., Lagarrigue S., Peiniau P., Hermier D., 2000. Role of hepatic lipogenesis in the susceptibility to fatty liver in the goose (Anser anser). Comp. Biochem. Physiol. B Comp. Biochem., 126, 81-87.

Olver M.D., Preez J.J., Kuyper M.A., Mould D.J., 1977. The carcass composition and growth of the "mule" ducklings compared to purebred ducklings. Agroanimalia, 9, 7-12.

Onishi N. et al., 1955. Studies on the artificial inseminations of ducks. Bull. Nat. Inst. Agric. Sci., 11, 1-16.
Pé M.P., 2004. Principaux éléments du marché du foie gras. 6e Journ. Rech. Palmipèdes à Foie Gras, Arcachon, France, 7-8 Oct. 2004, 19-22.

Pingel H., Tittman G., Klemm R., 1989. Fertility and hatchability in crossing muscovy drakes and Pekin ducks. Proc. $8^{\text {th }}$ Int. Symp. Waterfowl, Budapest, Hongrie, 48-50.

Pingel H., 1990a. Genetics of egg production and reproduction in waterfowl. In: Poultry breeding and genetics, R.D. Crawford Ed., Elsevier, Amsterdam, Netherlands, 771-780.

Pingel H., 1990b. Genetics of growth and meat production in waterfowl. In: Poultry breeding and genetics, R.D. Crawford Ed., Elsevier, Amsterdam, Netherlands, 691-704.

Poivey JP, Cheng YS, Rouvier R., Tai C., Wang CT, Liu HL, 2001. Genetic parameters of reproductive traits in Brown Tsaiya ducks artificially inseminated with semen from Muscovy ducks. Poult. Sci., 80, 703-709.

Poujardieu B., Guichard F., Laventure P., 1994. Paramètres génétiques de croissance et de gavage de la cane commune. Genet. Sel. Evol., 26, 463-472.

Powell J.C., 1984. Selection for improved feed conversion ratio in the domestic ducks. Proc. $17^{\text {th }}$ World Poultry Cong., Helsinki, Finlande, 108-109.

Ramirez D.A., Arboleda C.R., Escote L.J., Ticohermonso E., 1976. The cytogenetics of intergeneric hybrids of Anas bochas and Cairina moschata. Phillipine Agriculturalist, 60, 6.

Retailleau B., 1999. Comparison of the growth and body composition of 3 types of ducks:pekin,Muscovy and Mule. Proc. $1^{\text {st }}$ World Conf. Waterfowl, Taichung, Taiwan, 1-4 Déc. 1999, 597-602.

Ricard F.H., de Carville H., Marché G.,1988. Etude comparative de la composition anatomique des canards de Barbarie, Pékin et mulard. In : La génétique du canard de Barbarie (Cairina moschata) et du mulard, R. Rouvier (ed.), Les colloques de l'INRA, 42, 75-99.

Robin N., Larroudé P., Peyhorgue A., Castaing J., 2002. Etude comparative de quatre types génétiques pour une production de canards lourds. 5e Journ. Rech. Palmipèdes à Foie Gras, Pau, France, 9-10 Oct. 2002, 41-45.

Robin N., Babilé R., Peyhorgue A., Dubois J.P., Leprettre S., 2004. Facteurs de production et qualité des foies gras de canards et d'oies. 6 emes Journ. Rech. Palmipèdes à Foie Gras, Arcachon, France, 7-8 Oct. 2004, 157-165.

Rouvier R., Tai J.J.L., Tai C., 1984. L'insémination artificielle des canes communes pour la production de mulards à Taïwan. La situation actuelle. In : Insémination artificielles et amélioration génétique : bilan et perspectives critiques. Les colloques de l'INRA, 29, 360-367.

Rouvier R., Mialon M.M., Salzmann F., Poujardieu B., 1988. Fertilité et éclosabilité des œufs d'une souche de cane Pékin (Anas platyrhynchos) en croisement interspécifique avec le Barbarie (Cairina moschata) par insémination artificielle. Ann. Zootech., 37, 73-86.

Rouvier R., Guy G., Rousselot-Paillet D., Poujardieu B., 1994. Genetic parameters from factorial cross breeding in two duck strain (Anas platyrhynchos) brown Tsaiya and Pekin, for growth and fatty liver traits. Br. Poultry Sci., 35, 509-517.

Salichon M.R., Guy G., Rousselot D., Blum J.C., 1993. Composition de trois types de foies 
oie, canard mulard et canard de barbarie. 1 ère Journ. Rech. Palmipèdes à Foie Gras, Bordeaux, France, 28-29 Avril 1993, 13-21.

Sauveur B., 1988. Reproduction des volailles et production d'oeufs. Editions INRA, Paris, France, $449 \mathrm{p}$.

Sellier N., Brun J.M., Richard M.M., Batellier F., Dupuy V., Brillard J.P., 2005. Comparison of fertility and embryo mortality following artificial insemination of common duck females (Anas platyrhynchos) with semen from common or Muscovy (Cairina moschata) drakes. Theriogenology, 64, 429-439.

Setiawan I, Babilé R, Auvergne A, et al. 1994. Growth and carcass composition of 2 genetic types in mule ducklings. Ann. Zootech., 43, 185196.

Shetty S., Griffin D.K., Graves J.A.M. 1999. Comparative painting reveals strong chromosome homology over 80 million years of bird evolution. Chrom. Res., 7, 289-295.

Snapir N., Rulf J., Meltzer A., Gvaryahu G., Rozenboim I., Robinzon B., 1998. Testosterone concentrations, testes weight and morphology of mule drakes (Muscovy drake $x$ Khaki Campbell). Br. Poultry Sci., 39, 572-574.

Stai S. M., Hughes C. R. 2003. Characterization of microsatellite loci in wild and domestic
Muscovy ducks (Cairina moschata). Anim. Genet., 34, 387-389.

Steele M.G., Wishart G.J., 1992. Evidence for a species-specific barrier to sperm transport within the vagina of the chicken hen Theriogenology, 38, 1107-1114.

Tai C., Rouvier R., 1998. Crossbreeding effect on sexual dimorphism of body weight in intergeneric hybrids obtained between Muscovy and Pekin duck. Genet. Sel. Evol., 30, 163-170.

Tai Liu J.J., Tai C., 1984. Studies on the artificial insemination of ducks. 3. A comparison of fertility of pooled semen and individual male semen in the crosses between Muscovy (Cairina moschata) and Tsaiya duck (Anas platyrhynchos var domestica). J. Taiwan Livest. Res., 17, 85-89.

Tai Liu J.J., Tai C., 1991. Mule duck production in Taiwan. I. Artificial insemination of ducks. Food and Fertilizer Technology Center, extension Bulletin, 328, 1-6.

Tai C., Poivey J.P., Rouvier R., 1994. Heritability for duration of fertility traits in Brown Tsaiya female ducks (Anas platyrhynchos) by artifical insemination with pooled Muscovy (Cairina moschata) semen. Br. Poult. Sci., 37, 571-577.

Takahashi H., Satoh M., Minezawa M., Purwadaria T., Prasetyo H., 2001. Characteriza- tion of duck microsatellite repeat sequences. Jap. Agric. Res. Quart., 35, 217-219.

Tchelycheva L.A., Delpech P., Popescu C.P., Rodionov A.V., 1993. Arrêt de la condensation chromosomique chez l'hybride interspécifique entre cane commune et canard de Barbarie, le mulard. Ann. Génét., 36, 190-193.

Tesoro, M., Migliore, L., Romboli, I.. 1986. The karyotype of a duck hybrid: Anas platyrhynchos x Cairina moschata. Ann. Fac. Med.Vet.di Pisa, 39, 153-158.

Velez A., Brun J.M., Rouvier R., 1996. Crossbreeding effects on reproductive traits in two strains of duck (Anas platyrhynchos): Brown Tsaiya and Pekin. Br. Poult. Sci., 37, 571-577.

Vinoo, R.; Thangaraju, P.; Nainar, A. M. 1999. Karyological studies on domestic fowl, Japanese quail and domestic duck using embryo culture technique. Ind. J. Poultry Sci., 34, 135-139.

Watanabe M., 1959. Partial infertility of intergeneric hybrid eggs between the Muscovy Drake and the Common duck. J. Fac. Fish. Anim. Husb., Hiroshima Univ., 2, 330-373.

Watanabe M., 1961. Experimental studies on the artificial insemination of domestic ducks, with special references to the production of the mule ducks. J. Fac. Fish Anim. Husb., Hiroshima Univ., 3, 439-485.

\section{Résumé}

Le mulard, qui assure plus de $90 \%$ de la production française de foie gras, est un hybride intergénérique entre le canard de Barbarie et la cane commune. L'hybride réciproque (canard commun $x$ cane Barbarie) ne présente pas d'intérêt zootechnique. Depuis les années 80, le mulard est obtenu par insémination artificielle, avec un taux de fertilité voisin de $\mathbf{7 0} \%$ à condition d'inséminer les canes deux fois par semaine à cause de la courte durée de la période fertile en hybridation. L'article envisage d'abord les aspects zoologiques de cette hybridation (cytogénétique, accouplement, fertilisation et développement embryonnaire, stérilité des hybrides). Il aborde ensuite, d'un point de vue zootechnique, les performances de reproduction de la cane commune mère du mulard (ponte, fertilité et durée de la période fertile, éclosabilité) et les caractères du mulard (croissance, couleur du plumage, comportement, efficacité alimentaire, composition corporelle, aptitude au gavage et qualité des produits). L'objectif est d'abord de décrire les spécificités de cet hybride relativement aux espèces parentales et lorsque c'est possible (présence de l'hybride réciproque), d'interpréter ses performances en fonction des contributions parentales. Il s'agit ensuite de réaliser une synthèse sur les estimées des paramètres génétiques des caractères des mulards dans les populations parentales et des corrélations génétiques entre performances en pur et en croisement. Ces paramètres conditionnent les possibilités d'amélioration du mulard par sélection génétique dans ces souches. Enfin, sont présentés des éléments de génétique moléculaire (marqueurs génétiques, cartographie comparée).

\section{Abstract}

\section{The mule duck: genetic determinism of an intergeneric hybrid}

The mule duck, an inter-generic hybrid between the Muscovy drake and the common duck, produces more than $90 \%$ of the French "foie gras". The reciprocal hybrid (common drake x Muscovy female duck) offers no interest in animal breeding. Since the 1980's, the mule duck has been obtained by artificial insemination, with a fertility rate reaching $70 \%$ owing to artificial insemination practised twice a week, because of the short duration of the fertile period in the crossbreeding. Thise synthesis first deals with the zoological aspects of this hybridisation (cytogenetics, mating, fertilisation and embryonic development, hybrid sterility). Then, it deals with, from a zootechnical point of view, the reproductive performances of the common duck female (laying, fertility and duration of fertility, hatchability) and the traits of the mule duck (growth, plumage colouration, behaviour, feed efficiency, body composition, force feeding, quality of the products). The aim was first to describe the specificity of the mule duck with respect to the parental species, and, when possible (the presence of the reciprocal hybrid), to interpret its performances in terms of parental genetic contributions. Another aim was to review the genetic parameters of mule traits estimated within the parental strains and the genetic correlations between purebred and crossbred performances. These parameters determine the potential of within-strain selection to improve the mule duck. At last, some elements of molecular genetics are presented (genetic markers, compared genetic maps).

BRUN J.-M., RICHARD M.-M., MARIE-ETANCELIN C., ROUVIER R., LARZUL C., 2005. Le canard mulard : déterminisme génétique d'un hybride intergénérique. INRA Prod. Anim., 18, 295-308. 